Ann. Sci. forest., 1969, 26 (2), 183-224.

\title{
CONDITIONS FORESTIÈRES ET POTENTIALITÉ DE L'ÉPICÉA EN HAUTE ARDĖCHE
}

\author{
H. OSWALD
}

avec la collaboration technique de A. Divoux, F. Michaux et A. Tisserand

Station de Sylviculture et de Production, Centre national de Recherches forestières, 54-Nancy Institut national de la Recherche agronomique

\section{SOMMAIRF}

La présente étude concerne la variation de la production de l'Épicéa (Picéa abies L.) en fonction du milieu sur les hauts plateaux de r'Ardèche et de la Haute-Loire - au-dessus de $1000 \mathrm{~m}$ d'altitude — dans le Massif Central.

Plus de 40 peuplements et plantations (placettes temporaires avec analyse de tiges) ont été étudiés, par des méthodes de régressions multiples. Parmi les facteurs analysés en détail, comme laltitude, la roche mère, l'exposition, la pente, etc., laltitude à elle seule explique environ $87 \%$ de la variation de la hauteur dominante des peuplements à 75 ans.

Les résultats obtenus permettent de confirmer la production très élevée de l'essence en cause et de fixer une limite altitudinale supérieure ( 1400 à $1450 \mathrm{~m}$ ) pour un reboisement rentable, dans les conditions actuelles. Des observations ont pu être fournies sur la sylviculture régionale de l'Épicéa.

\section{INTRODUCTION}

Ces dernières années, plusieurs études des potentialités agricoles furent entreprises dans diverses régions de la France, et, il est apparu qu'il était souhaitable d'étudier conjointement les potentialités forestières pour évaluer les ressources d'une région, base de planification et d'aménagement (s.C.A.F.R., 1965; GADANT, 1968a, 1968b). Il ne peut être question de traiter ici tous les avantages directs et indirects à caractère biologique, physique ou social que la forêt procure à l'homme, et dont l'importance quantitative est souvent difficile à chiffrer, mais il 
faut souligner certaines fonctions de la forêt dans la vie rurale et nous citons notamment parmi les rôles qu'elle joue :

- production de bois et de produits accessoires ;

— support d'activités économiques pour les populations voisines ;

- eaisse d'épargne * pour fournir occasionnellement des capitaux pour financer un investissement ou compenser un revenu défaillant.

Dans les régions ayant une économie rurale précaire, la forêt ne saurait que favoriser la rénovation et le maintien d'une vie rurale.

Un des objectifs majeurs est la recherche d'une évolution optimale pour l'utilisation des terres dans un cadre régional et, il faut se garder d'attribuer à la forêt les seules terres inaptes à l'agriculture.

C'est à la suite d'une étude sur les potentialités agricoles en Haute-Ardèche de J.P. Deffontaines et J. Bougler (1965), que la Station de Sylviculture et de Production du CNRF a entrepris des recherches sur les potentialités forestières dans la même région.

\section{TABLEAU 1}

J.P. Dfffontalnes (1967) - Occupation actuelle des terrains

\begin{tabular}{|c|c|c|}
\hline \multirow{2}{*}{ Type d'occupation } & \multicolumn{2}{|c|}{ Surface } \\
\hline & Hectares & $\%$ \\
\hline 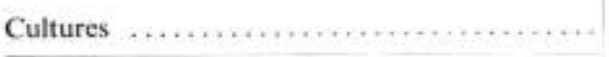 & 7943,8 & 16,8 \\
\hline 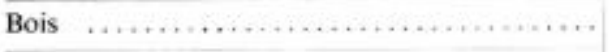 & 11983.4 & 25,6 \\
\hline 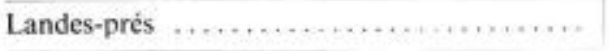 & 6126,1 & 13,0 \\
\hline Landes boisces $\ldots \ldots \ldots \ldots \ldots \ldots \ldots \ldots \ldots \ldots$ & 21004,7 & 44,6 \\
\hline Total .............. & 47058,0 & 100,0 \\
\hline
\end{tabular}

TABLEAU 2

J.P. Deffontaines (1967) - Classement des terrains

\begin{tabular}{|c|c|c|c|}
\hline \multirow{2}{*}{ Terrain } & \multicolumn{2}{|c|}{ Surface } & \multirow{2}{*}{ Critères de discrimination } \\
\hline & Hectares & $\%$ & \\
\hline T $1 \ldots$. & 9972,0 & 21,2 & $\begin{array}{l}\text { Profondeur du sol supérieure à } 25 \mathrm{~cm} \text {, pente inférieure } \\
\text { à } 15 \% \text {, texture fine }\end{array}$ \\
\hline T $2 \ldots$ & 3975,2 & 8,4 & $\begin{array}{l}\text { Profondeur inféricure à } 25 \mathrm{~cm} \text { ou à texture grossière, } \\
\text { pente inférieure à } 15 \%\end{array}$ \\
\hline T $3 \ldots \ldots \ldots$ & 2511,6 & 5,3 & Terrains présentant un excès d'eau permanent \\
\hline T $4 \ldots \ldots \ldots$ & 30599.2 & 65,1 & Terrains à pente supérieure à $15 \%$ \\
\hline Total ..... & 47058,0 & 100,0 & \\
\hline
\end{tabular}


Dans la revue \& Fourrages $>$ J.P. DefFontaines (1967) expose ses méthodes originales pour l'estimation des potentialités agricoles, leurs intérêts et leurs limites.

Dans les tableaux 1 et 2 , nous donnons un certain nombre de résultats de cette étude qui a porté sur 20 communes d'une superficie totale de 47058 ha.

Compte tenu d'une éventuelle mise en valeur (défrichement, assainissement, etc.), il reste environ 21600 ha sur des terrains T4 à reboiser, ce qui donnerait une surface de 32000 ha de forêt et un taux de boisement d'environ $68 \%$.

La nécessité de reboiser certains terrains du Massif Central avait déjà été évoquée au début du $19^{\circ}$ siècle, et la fameuse lettre du Comte de MontLosier au Préfet du Puy-de-Dôme, écrite le 18 février 1827 (J.O. 1955) est pleine d'intérêt et toujours d'actualité ; elle touche au fond du problème foncier de cette région. Mais il a fallu attendre les années 1860 à 1910 , période * héroïque * de reboisement et de restauration des terrains en montagne, en France comme en Europe centrale, pour que de grands travaux de reboisement fussent entrepris dans le Massif Central. La plupart des peuplements d'Épicéa que nous avons étudiés datent de cette époque.

La période de 1910 , jusqu'à la fin de la deuxième guerre mondiale, a été marquée par un ralentissement sensible des travaux de reboisement; ce n'est qu’après la création du Fond Forestier National, en 1946, et plus spécialement depuis 1952, que les reboisements ont pris un essort spectaculaire. Les régions naturelles comme le Velay basaltique, la Margeride et le plateau du Haut et Moyen Vivarais furent retenues, entre autres, comme zones prioritaires. L'évolution actuelle de l'agriculture laisse prévoir que le Massif Central pourrait devenir une des plus importantes régions forestières de France (J. PARDÉ, 1966 a).

\section{1 - GENERALITES}

\section{1. - Situation géographique et limites}

S'tuée au sud-est du Massif Central, la région étudiée est centrée sur la Haute-Ardèche, mais elle s'éiend quelque peu au nord, nord-ouest, et ouest sur le département de la Haute-Loire.

Le périmètre de la région (fig. 1) est constitué :

- au nord, par une ligne, Le Puy - Mt-Mézenc (1 754 m) - Mézilhac

- à l'est, par une ligne, Mézilhac - Massif du Tanargue (1 $441 \mathrm{~m}$ )

- au sud, par une ligne, Massif du Tanargue - Le Trepalour (1 $408 \mathrm{~m}$ ) jusqu'à la vallée de l'Allier ; il limite une superficie de 130000 ha à l'exclusion toutefois des zones à altitude inférieure à $1000 \mathrm{~m}$ comme les fonds de vallées de l'Ardèche, de l'Allier et de la Loire. 


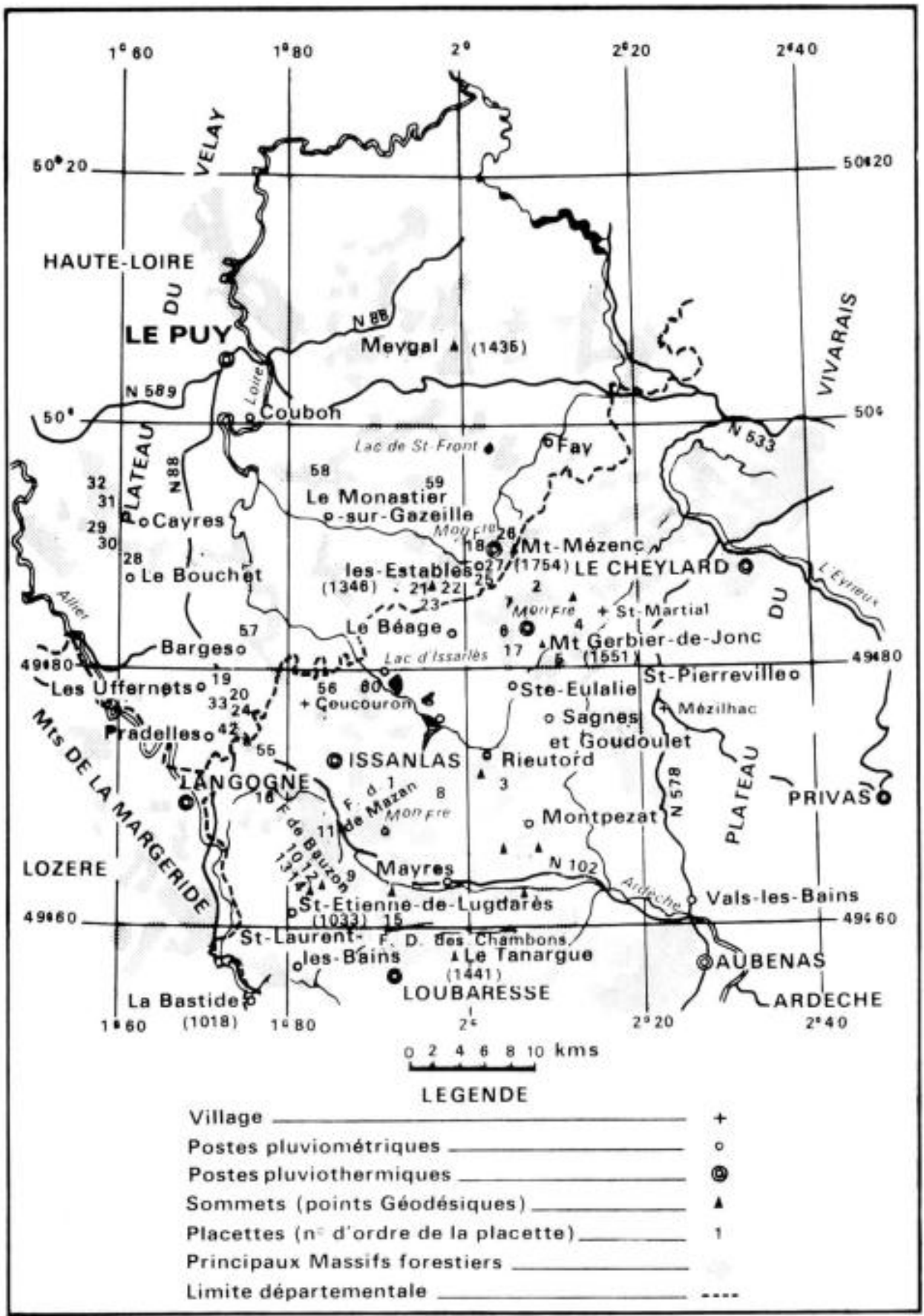

FIG. 1. - Délimitation de la région, répartition des placettes et emplacements des postes météorologiques 
Ce périmètre englobe en partie deux régions naturelles: le Vivarais et le Velay, le point culminant est le Mt-Mézenc $(1754 \mathrm{~m})$. Dans l'est de la région, dans les monts du Vivarais, le relief est tourmenté et les rebords orientaux des hauts plateaux, sur la ligne de partage des eaux Méditerranée-Atlantique, sont puissamment attaqués par l'érosion. Tous les affluents du Rhône (l'Ardèche et l'Eyrieux) ont un régime torrentiel et s'enfoncent rapidement dans des vallées étroites et récentes. Le centre et l'ouest de la région sont caractérisés par une succession de hauts plateaux entre $1100 \mathrm{~m}$ et $1400 \mathrm{~m}$ d'altitude, entrecoupés par les vallées anciennes de la Loire, de l'Allier et de leurs affluents. Le relief est beaucoup moins prononcé et l'érosion actuelle est très faible.

\section{2. - GÉologie ET DESCRIPTION DES ROCHES}

Le socle primaire hercynien est essentiellement constitué de roches granitiques ou cristallophylliennes et formait une pénéplaine au début du tertiaire. A la suite du plissement alpin, dès l'Oligocène, se manifesta une activité volcanique qui dura jusqu'au quaternaire, et l'homme a certainement été témoin des dernières éruptions (L. MORET, 1958). La majorité des basaltes et phonolites de la région date du Pliocène supérieur.

\section{Description sommaire des principales roches de la région}

1) granite et granite porphyrö̈de (souvent chargés de débris de gneiss)

2) granite gneissique

3) gneiss rubanné et micaschistes

4) une série de basaltes, d'âges différents, y compris les labradorites. Les basaltes du Pliocène supérieur sont les plus répandus, ils sont moins compacts et d'une couleur moins sombre

5) la phonolite (y compris les trachytes et andésites qui sont très peu répandus), est riche en silice et alumine, et pauvre en éléments ferromagnésiens. Elle se clive facilement en plaquettes de taille moyenne, forme des dykes ou sucs entourés d'importants éboulis, et constitue les principaux sommets au nord de la région.

Dans notre étude, nous ne distinguons que 3 roches mères : schistes,

1) Substratum granitique (G) comportant les granites, les gneiss et mica-

2) Substratum basaltique (B) constitué par les basaltes et labradorites,

3) Substratum phonolitique $(\mathrm{Ph})$ composé par les phonolites, les trachytes et andésites.

\section{3. - Altération des roches et types de sols}

L'étude des sols fut menée en étroite collaboration avec Monsieur F. LE TACON de la Station de Recherches sur les Sols forestiers du CNRF. Il a 
assuré l'analyse et l'interprétation des résultats, ainsi qu'une grande partie des relevés. Une étude détaillée est sous presse (LE TACoN F. et Oswald H., 1969).

L'altération des roches cristallines donne dans un premier stade un \&gore * qui, par la suite, évolue par des effets divers (cryoturbation, solifluction, colluvionnement) vers une arène dont l'épaisseur peut atteindre plusieurs mètres. Nous pouvons distinguer trois type de sols sur roche mère cristalline suivant l'altitude :

$1^{\circ}$ ) Ranker cryptopodzolique à moder pseudoalpin au-dessus de $1500 \mathrm{~m}$ d'altitude,

$2^{\circ}$ ) Sol brun cryptopodzolique à moder pseudoalpin (1 300 à $1500 \mathrm{~m}$ d'altitude),

$3^{\circ}$ ) Sol brun cryptopodzolique à moder au-dessous de $1300 \mathrm{~m}$ d'altitude.

Tous ces sols sont caractérisés par une granulométrie assez grossière et par une proportion de sable variant de 35 à $40 \%$; ils sont profonds et meubles (sauf certains rankers), leur $\mathrm{pH}$ est inférieur à 5 , leur taux de saturation est faible. dépassant rarement $10 \%$ sauf pour les horizons (B)/C.

Les teneurs en potassium et calcium échangeables sont faibles et même insuffisantes pour les rankers, à l'exception toutefois des horizons A ; par contre, les ceneurs en phosphore assimilable, sont toujours remarquablement élevées.

Les roches volcaniques, parmi lesquelles les phonolites, trachytes et andésites, forment un groupe distinct, évoluent d'une manière différente suivant qu'elles se présentent sous la forme de dykes, de dalles épaisses, de scories ou de cendres. Elles sont généralement moins altérées que les roches cristallines.

Nous pouvons distinguer deux types de sols sur roche mère volcanique :

1) Andosols oligotrophes humifères d'altitude, développés sur phonolites ;

2) Andosols mésotrophes formés à plus basse altitude sur produits basaltiques.

Morphologiquement, tous les sols dérivés de produits volcaniques que nous avons rencontrés ont des caractéristiques voisines. Le profil est de type $\mathrm{A} / \mathrm{C}$, l'incorporation de matière organique est toujours très forte, même si visuellement, elle parait moindre pour les sols de plus basse altitude. La texture au toucher est limoneuse, mais très particulière (toucher savonneux, glissant). En période sèche, ces sols sont pulvérulents. Les quelques mesures de densité apparente que nous avons effectuées donnent des valeurs voisines de 0,9 .

Les détermination de texture, effectuées après dispersion à l'hexamétaphosphate de sodium, donc probablement insuffisante, donnent une majorite d'éléments limoneux et une teneur en argile de 15 à $20 \%$. La teneur en sable est très faible (moins de $5 \%$ ) pour les sols issus de phonolite, plus variable pour les sols issus de produits basaltiques. Elle peut devenir importante sur scories (30 à $40 \%$ ).

Un des critères les plus importants, pour la caractérisation des andosols, est la présence d'allophane, qu'il est possible de doser sous forme d'alumine et de silice amorphe. Les dosages de ces éléments, effectués par le réactif combiné : acide oxalique, oxalate d'amonium et hydrosulfite de sodium (Duchaufour et 


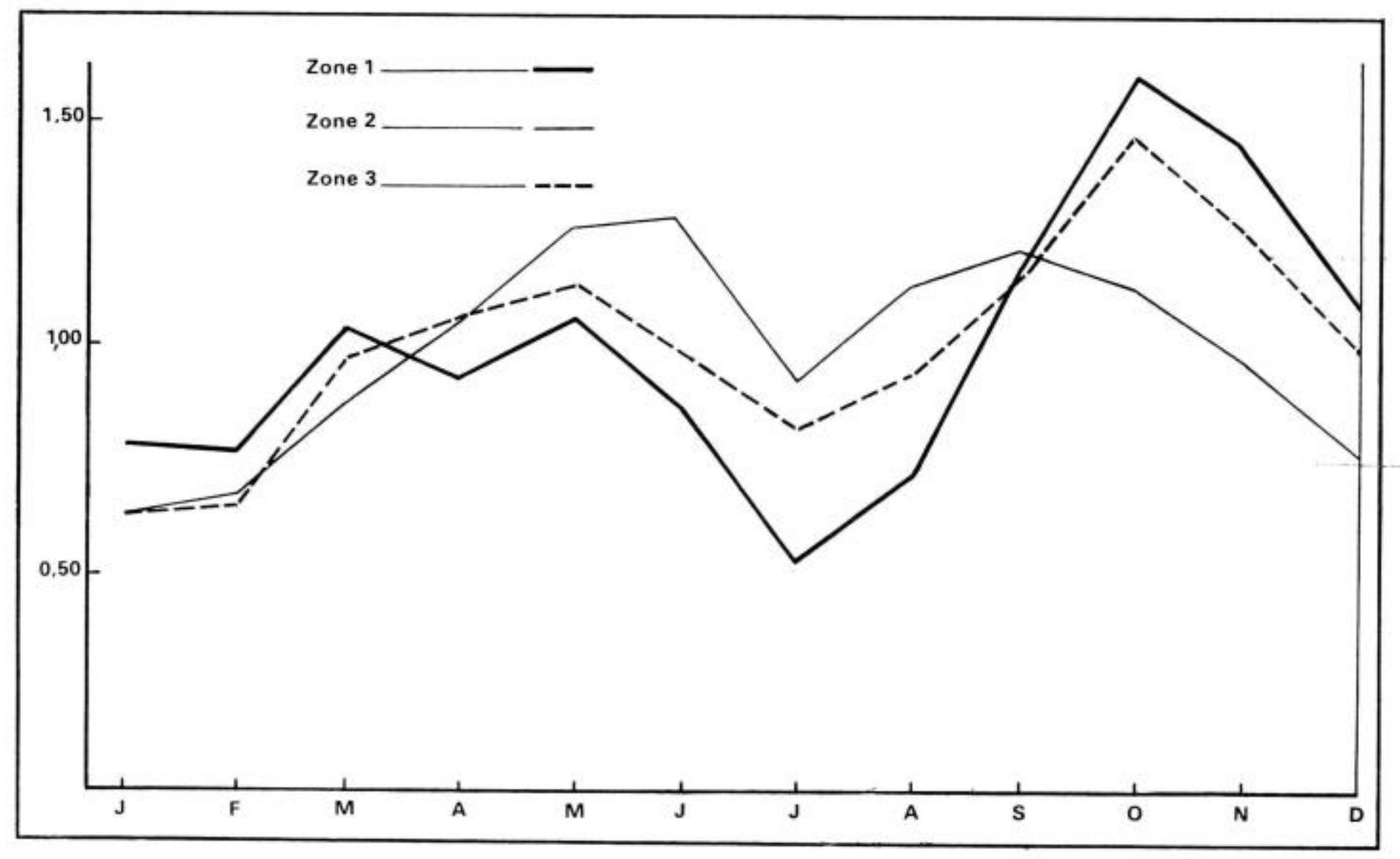

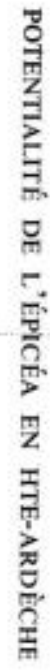

FIG. 2. - Fractions pluviométriques pour les trois zones d'après les valeurs normales des précipitations 
SOUCHIER, 1966), autorisent le classement de ces sols développés sur roche mère volcanique parmi les andosols, ou au moins de leur attribuer le terme andosolique.

\section{4 - Le Climat \\ 1.41. - Introduction}

Les études sur les climats du Massif Central sont assez nombreuses et parfois très détaillées; mais elles ne permettent pas une extrapolation à l'échelle de la station. Nous nous limiterons à une description générale des différents climats ; par contre, nous allons étudier d'une manière plus approfondie les corrélations existantes entre certains facteurs du climat et l'altitude. Les données dont nous disposons sont essentiellement tirées des publications de ronM pour les différentes périodes ; mais malheureusement, il n'était pas toujours possible de prendre les mêmes périodes et les mêmes stations en considération pour l'étude des climats. La figure 1 donne les emplacements des postes météorologiques de la région.

\subsection{2. - Les précipitations}

Nous disposons d'un ensemble de 30 stations pluviométriques dans la région, dont un certain nombre furent installées par l'EDF depuis 1953. Nous avons particulièrement étudié la quantité totale des précipitations, la part de la neige, le régime mensuel et leurs variations ainsi que leur répartition géographique et altitudinale.

\subsection{Le régime mensuel}

A partir de 25 stations - la plupart des données peuvent être considérées comme des normales - nous avons calculé les fractions pluviométriques (1) d'après P. EstienNe (1956) et on peut en déduire trois zones à régime pluviométrique différent (fig. 2).

Une première zone au sud, sud-est et à l'est de la région, se distingue par un minimum de précipitations très prononcé au mois de juillet, par un maximum très élevé en octobre, par un total de précipitations annuelles important (1 $284 \mathrm{~mm}$ ) et par une intensité considérable des pluies. Ces caractéristiques correspondent au climat cévénol pour lequel l'influence méditerranéenne, avec ses fortes variations, est très marquée (P. ESTIENNE, 1956).

La deuxième zone est située dans la partie nord-ouest de la région, sur les plateaux du Devès, entre les vallées de la Loire et de l'Allier. Elle a un climat à influence continentale. Les précipitations annuelles sont significativement plus faibles (749 mm) ainsi que leur intensité ; il existe un minimum en hiver (janvier) et un maximum en été. La variation mensuelle de l'indice pluviométrique est, dans son ensemble, moins importante.

(1) Rapport de la pluviosité moyenne du mois à la pluviosité du mois moyen $(1$ mois $=30$ jours). 
La troisième zone enfin, qui occupe le centre de notre région, est caractérisée par un climat de transition où l'influence méditerranéenne devient moins marquée La moyenne annuelle est encore élevée $(1066 \mathrm{~mm})$ ainsi que le maximum en octobre ; par contre le minimum en juillet est moins prononcé et l'intensité des pluies reste intermédiaire.

Le tableau 3 donne, à titre de comparaison, un certain nombre de résultats pour ces trois zones.

\subsection{Les précipitations annuelles et leurs variations}

Tous les auteurs, et notamment P. Estienne (1956), font remarquer la grande variabilité du climat du Massif Central. Pour l'ensemble des 30 stations pluviométriques, nous avons pu constater qu'il existe, outre les différences entre les trois zones, une très grande variation des précipitations d'année en année. Dans le tableau 3, nous avons noté le maximum et le minimum des précipitations annuelles pour la période de 1956 à 1964. Si les valeurs moyennes sont relativement élevées, des années très sèches sont toujours à craindre et peuvent avoir des incidences sur la croissance des végétaux.

Le nombre moyen de jours de précipitations par année varie pour la même période de 135 jours (minimum : le Bouchet-St-Nicolas, zone 2) à 203 jours (Lac d'Issarlès, zone 1). Les jours de pluie sont relativement bien répartis sur tous les mois. Les orages sont très fréquents et parfois d'une intensité considérable.

\subsection{La neige}

Les précipitations neigeuses ont une action importante sur la végétation ct sur le sol. Le manteau neigeux protège le sol et la végétation basse contre des gelées excessives et permet une alimentation en eau même en hiver, ce qui peut être vital pour les arbres ; il constitue une réserve d'eau importante et offre une protection efficace contre les effets mécaniques du vent.

Mais l'effet de la neige est souvent néfaste pour les arbres. Nous citons notamment les énormes dégâts causés à la forêt pendant l'hiver 1957/58 où des peuplements entiers furent brisés sous la charge d'une masse importante de neige lourde et humide dans toute la région et surtout en Forêt domaniale de Mazan (L. GAIlLARD, 1964). S'il s'agissait là d'une véritable catastrophe, d'importantes quantités de chablis sont à déplorer chaque hiver et nous avons pu constater que rares sont les arbres dans nos placettes dont la cime n'avait pas été brisée au moins une fois durant leur existence, et ceci semble indépendant de l'altitude et de l'exposition.

Le nombre de jours de précipitations neigeuses annuel pour la période 1956/64 (tabl. 3) varie entre 22 (minimum du Puy) et 63 (maximum d'Issanlas) ; il est d'environ 50 jours en moyenne, mais on constate, même en plein hiver et en altitude, une alternance des chutes de neige et de pluie et, de ce fait, une grande fragilité du manteau neigeux qui ne persiste pas dans son intégralité tout l'hiver. D'importantes chutes de neige, des tempêtes de neige et surtout la formation d'énormes congères rendent toute circulation difficile et parfois même impossible. 


\section{TABLEAU 3}

Pluviométrie des 3 zones ( 3 stations) pour la période 1956-1964 d'après les données du Bulletin pluviométrique mensuel de la Météorologie nationale

\begin{tabular}{|c|c|c|c|c|c|c|c|c|c|c|c|c|c|c|c|c|c|}
\hline Station & & Période & J & $\mathrm{F}$ & $\mathrm{M}$ & A & $\mathrm{M}$ & J & J & A & $\mathrm{S}$ & O & $\mathrm{N}$ & D & Total & Max. & Min. \\
\hline \multirow{5}{*}{$\begin{array}{l}\text { Issanlas } \\
\quad \text { Altitude : } 1220 \mathrm{~m}\end{array}$} & 1 & $1956 / 64$ & 75,0 & 75,7 & 124,6 & 134,5 & 108,4 & 107,6 & 67,8 & 85,7 & 115,8 & 140,0 & 146,2 & 126,2 & 1307,5 & 1568,9 & 899,4 \\
\hline & 2 & $1956 / 64$ & 24,0 & 19,4 & 23,5 & 43,1 & 33,6 & 30,1 & 22,9 & 22,7 & 45,2 & 44,7 & 59,9 & 39,7 & - & 135,5 & - \\
\hline & 3 & $1956 / 64$ & 19,2 & 15,7 & 17,8 & 17,2 & 14,1 & 13,9 & 11,8 & 11,7 & 11.7 & 17,9 & 18,2 & 20,7 & 189,9 & 220 & 167 \\
\hline & 4 & $1956 / 64$ & 13,8 & 11,3 & 8,9 & 6,8 & 1,0 & 0 & 0 & 0 & 0 & 3,0 & 7,0 & 11,6 & 63,4 & 85 & 35 \\
\hline & 5 & $1951 / 60$ & 22 & 22 & 13 & 5 & 1 & 0 & 0 & 0 & 0 & 1 & 5 & 12 & 81 & - & - \\
\hline \multirow{5}{*}{$\begin{array}{l}\text { Le Bouchet-Saint- } \\
\text { Nicolas } \\
\text { Altitude : } 1228 \mathrm{~m}\end{array}$} & 1 & $1956 / 64$ & 52,3 & 49,9 & 80,8 & 83,3 & 76,0 & 77,0 & 61,6 & 76,1 & 83,8 & 70,6 & 74,8 & 77,4 & 863,6 & 1073,4 & 579.3 \\
\hline & 2 & $1956 / 64$ & 16,3 & 14,0 & 25,1 & 23,7 & 27,5 & 20,2 & 17,6 & 26,9 & 30,7 & 24,7 & 31,5 & 25,7 & - & 76,0 & - \\
\hline & 3 & $1956 / 64$ & 12,0 & 10,2 & 13,0 & 13,2 & 10,6 & 10,6 & 9,7 & 9,4 & 8,8 & 11,6 & 12,4 & 13,3 & 134,8 & 167 & 111 \\
\hline & 4 & $1956 / 64$ & 8,8 & 6,3 & 5,4 & 6,1 & 0,7 & 0 & 0 & 0 & 0 & 2,7 & 4,9 & 8.3 & 43,2 & 62 & 25 \\
\hline & 5 & $1951 / 60$ & - & - & - & - & - & - & - & - & - & - & - & - & 66 & - & - \\
\hline \multirow{5}{*}{$\begin{array}{l}\text { Sainte-Eulalie } \\
\text { Altitude : } 1360 \mathrm{~m}\end{array}$} & 1 & $1956 / 64$ & 101,2 & 99,8 & 140,6 & 131,8 & 110.0 & 123.7 & 63,6 & 107,3 & 137.5 & 193.8 & 187,2 & 144,0 & 1540,5 & 2240,2 & 1141,8 \\
\hline & 2 & $1956 / 64$ & 39,6 & 31,1 & 39,5 & 38,6 & 44,9 & 36,8 & 23,4 & 45,7 & 66,0 & 66,3 & 67,9 & 55,1 & - & 179,0 & - \\
\hline & 3 & $1956 / 64$ & 14,3 & 11,6 & 13,3 & 14,6 & 10,7 & 11,8 & 10,2 & 10,3 & 9,6 & 12,6 & 14,7 & 15,8 & 149,5 & 169 & 130 \\
\hline & 4 & $1956 / 64$ & 9,8 & 7,4 & 6,6 & 4,6 & 0,1 & 0 & 0 & 0 & 0 & 2,8 & 5,4 & 11,4 & 48,1 & 60 & 27 \\
\hline & 5 & $1951 / 60^{\circ}$ & - & - & - & - & - & - & - & - & - & - & - & - & 75 & - & - \\
\hline
\end{tabular}

$1^{\text {re }}$ ligne : pricipitations moyennes mensuelies en mm.

$2^{2}$ ligne : intensité moyenne en $\mathrm{mm}$.

$3^{\text {e }}$ ligne : nombre total de jours de précipitations.

$4^{\text {e }}$ ligne : nombre de jours de précipitations neigeuses.

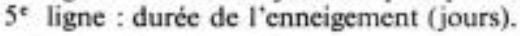

Estimation d'après BALSEINTE R. (1966), 
Il faut mentionner ici un phénomène dont l'importance écologique est considérable : c'est la formation de givre et de verglas sur les arbres. Le givre, qui est très fréquent pendant l'hiver surtout à des altitudes élevées, empêche d'une manière constante la dissémination des graines de l’épicéa et met ainsi en cause toute régénération naturelle de cette essence.

\subsection{Les précipitations et laltitude}

D'une manière générale, les précipitations augmentent avec l'altitude, atteignent un \& optimum pluvial s et peuvent décroître ensuite. Or, nous n'avons pu constater un gradient altitudinal que dans la zone 2 au NW de la région et l'explication peut être donnée partiellement par le relèvement général du niveau des nuages au-dessus des grands plateaux du Vivarais et du Velay. Par contre, on observe une augmentation du nombre de jours de précipitations neigeuses et de la durée du manteau nival avec l'altitude.

\subsection{3. - Les températures}

Dans la région concernée, nous ne disposons que de 9 stations d'observations de températures (fig. 1) qui ne sont malheureusement que partiellement comparables (différents types d'abris et différentes périodes d'observations); en plus on ne peut pas tenir compte de l'effet de l'exposition ni de lorographie. Dans le tableau 4, nous donnons les différentes valeurs mensuelles pour ces 9 stations, ainsi que, à titre de comparaison, celles de 2 stations sommitales (Mont Aigoual $1567 \mathrm{~m}$ et le Puy-de-Dôme $1465 \mathrm{~m}$ ), au sud et au nord de la région.

\subsection{Les températures mensuelles et annuelles et leurs variations avec l'altitude}

A partir de ces données, nous avons calculé le gradient altitudinal pour les températures moyennes mensuelles, dont les résultats figurent au tableau 5. La liaison température moyenne mensuelle (ii) en fonction de l'altitude est linéaire et de la forme :

$$
f i=a+b X
$$

$f i=$ température moyenne du ième mois ;

$a=$ terme constant qui correspond à la température réduite au niveau de la mer ;

$b=$ coefficient de régression ou gradient altitudinal du ième mois ;

$X=$ altitude (m) multipliée par $10^{-2}$.

Les coefficients de corrélation sont très élevés $(-0,922$ à $-0,997)$ et l'écart type résiduel varie entre $0,37{ }^{\circ} \mathrm{C}$ et $0,96{ }^{\circ} \mathrm{C}$ suivant le mois considéré.

La variation des gradients mensuels au cours de l'année est très prononcée, ce qui souligne le contraste de climat entre la Haute et la Basse Ardèche. Les gradients de température sont supérieurs aux valeurs trouvées par $\mathrm{Ph}$. DAGET et J. Poissonet (1966) pour le Massif Central et aux valeurs citées par Coutagne (1954) pour plusieurs massifs des Alpes. 
TABLEAU 4

Températures mensuelles moyennes, températures annuelles moyennes ( $i)$ maximales (iM), minimales (im), amplitude annuelle et période de végétation.

\begin{tabular}{|c|c|c|c|c|c|c|c|c|c|c|c|c|c|c|c|c|c|c|c|c|c|}
\hline \multirow[b]{2}{*}{ Stations } & \multirow[b]{2}{*}{ Alt. } & \multirow[b]{2}{*}{ Période } & \multirow[b]{2}{*}{$\mathbf{J}$} & \multirow[b]{2}{*}{$\mathrm{F}$} & \multirow[b]{2}{*}{ M } & \multirow[b]{2}{*}{ A } & \multirow[b]{2}{*}{ M } & \multirow[b]{2}{*}{ J } & \multirow[b]{2}{*}{ J } & \multirow[b]{2}{*}{ A } & \multirow[b]{2}{*}{$\mathrm{S}$} & \multirow[b]{2}{*}{ O } & \multirow[b]{2}{*}{$\mathrm{N}$} & \multirow[b]{2}{*}{ D } & \multirow{2}{*}{$\begin{array}{c}\bar{t} \\
\text { annee }\end{array}$} & \multirow{2}{*}{$\begin{array}{c}\text { tM } \\
\text { année }\end{array}$} & \multirow{2}{*}{ tm } & \multirow{2}{*}{ 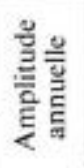 } & \multicolumn{3}{|c|}{ Période de végétation } \\
\hline & & & & & & & & & & & & & & & & & & & $\begin{array}{l}\text { Début } \\
\mathrm{N}^{\circ} \mathrm{du} \\
\text { jour }\end{array}$ & $\begin{array}{l}\text { Fin } \\
N^{\circ} d u \\
\text { jour }\end{array}$ & $\begin{array}{l}\text { Durée } \\
\text { jours }\end{array}$ \\
\hline Aubenas ...... & $240 \mathrm{~m}$ & $1930 / 49$ & 4,0 & 5,1 & 8,8 & 12,1 & 15,8 & 20,0 & 22,7 & 22,0 & 18,5 & 13,2 & 8.1 & 4,4 & 13,1 & 17,9 & 8,4 & 18,7 & 60,0 & 326,5 & 266,5 \\
\hline Privas ........ & $300 \mathrm{~m}$ & $1930 / 49$ & 3,3 & 4,9 & 8,7 & 12,4 & 15,3 & 19.4 & 22,2 & 21,3 & 18,0 & $\overline{12,8}$ & 7,8 & 3,8 & 12,5 & 17,2 & 7,7 & 18,9 & 61,5 & 323,5 & 262,0 \\
\hline Le Cheylard ... & $467 \mathrm{~m}$ & $1930 / 49$ & 2,3 & 4,4 & 7.5 & 10.9 & 14,1 & 17,9 & 21,3 & 20,8 & 17,4 & $\overline{11,9}$ & 7,1 & 3,2 & 11,6 & 17,4 & 5,6 & 19,0 & 69,5 & 319.5 & 250.0 \\
\hline Le Puy ....... & $714 \mathrm{~m}$ & $1921 / 50$ & 1,2 & 2,3 & 5,4 & 8,4 & 12,0 & 15,8 & 18,2 & 17,9 & 15,0 & 10,3 & 5,5 & 1.5 & 9,4 & 14,3 & 4,4 & 17.0 & 91,0 & 309,5 & 218,5 \\
\hline Langogne ..... & $927 \mathrm{~m}$ & $1950 / 60$ & $-0,5$ & $-0,5$ & 3,5 & 6,5 & 10,5 & 14,0 & 16.5 & 15,5 & 13,0 & 8.0 & 3,4 & 2,0 & 7,7 & 13,0 & 2,3 & 17,0 & 109,5 & 295,0 & 185,5 \\
\hline Loubaresse .... & $1220 \mathrm{~m}$ & $1955 / 63$ & $-0,5$ & $-0,1$ & 2,3 & 4,8 & 9.4 & 12,8 & 15,5 & 15,0 & 13,1 & 8,0 & 3,0 & 0,1 & 6,9 & - & - & 16,0 & 119,5 & 294,5 & 175.5 \\
\hline Issanlas ....... & $1220 \mathrm{~m}$ & $1955 / 63$ & $-1,3$ & $-1,1$ & 1,8 & 4,4 & 8,6 & 11.5 & 14,7 & 14,1 & 12,0 & 7,2 & 2,1 & 1,8 & 6,3 & - & - & 16,0 & 124,0 & 289.5 & 165,5 \\
\hline Sainte-Eulalie . & $1360 \mathrm{~m}$ & $1930 / 49$ & $-2,1$ & $-2,1$ & 1,0 & 3,8 & 7,3 & 11,5 & 13,9 & 14,0 & 10,5 & 5,2 & 1,5 & $-1,9$ & 5,3 & 9,8 & 0.7 & 16,1 & 133,0 & 278,0 & 145,0 \\
\hline Mont Mézenc.. & $1535 \mathrm{~m}$ & $1891 / 1930$ & $-2,3$ & $-1,8$ & $-0,5$ & 2,5 & 5,9 & $\overline{9,9}$ & 13,1 & 12,7 & 10,2 & 5.7 & 1,6 & $-2,1$ & 4,6 & - & - & 15,4 & 144,0 & 280,0 & 136,0 \\
\hline
\end{tabular}

TABLEAU 5

Les gradients thermiques mensuels pour les temperatures moyennes mensuelles

\begin{tabular}{|c|c|c|c|c|c|c|c|c|c|c|c|c|c|}
\hline $\begin{array}{c}\text { Température } \\
\text { moyenne } \\
\text { mensuelle }\end{array}$ & J & F & $\mathrm{M}$ & A & M & J & $\mathbf{J}$ & A & $\mathrm{S}$ & $\mathrm{O}$ & $\mathrm{N}$ & D & Annuel \\
\hline $\begin{array}{l}\text { Gradient thermi- } \\
\text { que }: \ldots \ldots \ldots b\end{array}$ & $-0,479$ & $-0,599$ & $-0,720$ & $-0,783$ & $-0,735$ & $-0,766$ & $-0,764$ & $-0,730$ & $-0,652$ & $-0,615$ & $-0,553$ & -0.448 & $-0,659$ \\
\hline Terme constant : $a$ & 4,699 & 6.540 & 10,658 & 14,256 & 17,505 & 21,542 & 24.336 & 23.501 & 19,969 & 14,594 & 9,371 & 5,390 & 14,445 \\
\hline $\begin{array}{l}\text { Coefficient de } \\
\text { corrélation :. } R\end{array}$ & $-0,983$ & $-0,970$ & $-0,997$ & $-0,996$ & $-0,995$ & -0.994 & $-0,991$ & -0.985 & $-0,983$ & $-0,979$ & $-0,985$ & $-0,922$ & $-0,994$ \\
\hline $\begin{array}{l}\text { Ecart-type rési- } \\
\text { duel }: \ldots \ldots, s\end{array}$ & 0,459 & 0,772 & 0,282 & 0,365 & 0,379 & 0,450 & 0,527 & 0,651 & 0.631 & 0,660 & 0,495 & 0,964 & 0.376 \\
\hline
\end{tabular}


Il faut cependant être très prudent pour l'interprétation des résultats : il s'agit d'estimations à partir d'un nombre de stations très limité et toute extrapolation en dehors de la zone considérée et des altitudes observées serait dangereuse.

Nous avons également calculé les gradiens altitudinaux pour les températures maximales et minimales, mais le nombre de stations était, dans ce cas, encore plus restreint et les corrélations, quoique linéaires, étaient moins étroites. Dans le tableau 6, nous donnons les moyennes des températures maximales et minimales pour Sainte-Eulalie (1 $360 \mathrm{~m}$ ).

\section{TABLEAU 6}

Températures maximales $(M)$ et minimales $(m)$ et amplitude $(a)$. Valeurs moyennes mensuelles de Sainte-Eulalie (I 360 m) (M.F. des Princes) pour la période do $1930 / 49$ d'après P. EsTneNNE (1956)

\begin{tabular}{|c|c|c|c|c|c|c|c|c|c|c|c|c|c|c|}
\hline Station & & J & $\mathbf{F}$ & M & A & M & $\mathbf{J}$ & J & A & $\mathrm{S}$ & $\mathrm{O}$ & $\mathbf{N}$ & D & Moyenne \\
\hline \multirow[t]{2}{*}{ Sainte-Eulalic } & M & 1,2 & 1,7 & 5,2 & 8,4 & 12,1 & 17,1 & 20,3 & 20.2 & 15.5 & 10,1 & 5,0 & 1,0 & 9,8 \\
\hline & $m$ & $-5,5$ & $-5,8$ & $-3,2$ & $-0,6$ & 2,5 & 5,7 & 7,4 & 7,8 & 5.5 & 0.2 & $-1,9$ & $-5,0$ & 0,7 \\
\hline Altitude : I $360 \mathrm{~m}$ & $a$ & 6,7 & 7.5 & 8,5 & 8,9 & 9.6 & 11,4 & 13,0 & 12,4 & 10,0 & 9,9 & 6,9 & 6,0 & 9,1 \\
\hline
\end{tabular}

Un autre phénomène bien connu (OzENDA, 1954) est la diminution de l'amplitude thermique annuelle avec l'altitude, c'est-à-dire, la différence entre la température moyenne du mois le plus chaud et celle du mois le plus froid. Cette diminution est également linéaire et nous avons obtenu comme équation :

$$
A=19,62-0,282 \mathrm{X} \quad R=-0,965, \quad s=0,39
$$

$A=$ amplitude thermique $\left(f_{7}-i_{1}\right)$

$X=$ altitude $(\mathrm{m}) \cdot 10^{-2}$

Le tableau 7 indique le nombre moyen mensuel des jours de gelées pour quatre stations d'après Estienne (1956) pour la période de 1940 à 1949. Nous citons encore à titre de comparaison le nombre total annuel des jours de gelées

\section{TABLEAU 7}

Nombre moyen mensuel des jours de gelées (pour la période 1940-49 d'après P. EsIIENNE (1956))

\begin{tabular}{|c|c|c|c|c|c|c|c|c|c|c|c|c|c|c|}
\hline Station & $\begin{array}{c}\text { Altitude } \\
\mathrm{m}\end{array}$ & J & $\mathrm{F}$ & M & A & $\mathrm{M}$ & $J$ & J & A & $\mathrm{S}$ & O & $\mathrm{N}$ & D & Total \\
\hline Privas $\ldots \ldots \ldots \ldots \ldots$ & 300 & 18,5 & 10,9 & 3,7 & 0,1 & 0,2 & - & - & - & - & 0,5 & 7,8 & 14,5 & 56,2 \\
\hline Le Puy .............. & 714 & 21,8 & 18,2 & 14,9 & 4,5 & 1,4 & - & - & - & - & 3,2 & 13,6 & 20,4 & 98,0 \\
\hline Sainte-Eulalie ....... & 1360 & 29,6 & 27,9 & 27,6 & 18,3 & 8,7 & 1,5 & 0,3 & - & 3,1 & $\overline{11,1}$ & 24,0 & 30,0 & 182,1 \\
\hline Mézenc $\quad \ldots \ldots \ldots \ldots$ & 1535 & 30,7 & 27,2 & 27,3 & 18,8 & 9.0 & 0,4 & 0,1 & 0,1 & 2,4 & 8,8 & 21,0 & 28,8 & 174,6 \\
\hline
\end{tabular}


pour Issarlès (151) et pour Loubaresse (117). Entre 1000 et $1300 \mathrm{~m}$ d'altitude, les mois de juillet et août seuls sont sans gelées, tandis que, à une altitude supérieure, des gelées peuvent apparaître à n'importe quelle époque de l'année. Des gelées tardives et précoces sont fréquentes au-dessus de $1000 \mathrm{~m}$ d'altitude.

\subsection{La période de végétation}

Il n'est pas nécessaire ici de souligner l'importance de la période de végétation; mais nous ne possédons pas d'observations phénologiques dans la région, du moins en ce qui concerne les essences forestières et plus spécialement l'épicéa, son débourrement, sa période de croissance en circonférence et en hauteur, sa fructification, etc. A cette fin, nous venons d'installer dans 4 peuplements d'épicéa, à différentes altitudes, un dispositif pour observer le déroulement de la croissance en circonférence à un niveau donné d'un tronc d'arbre. Parmi les méthodes conventionnelles, et plus ou moins empiriques, pour déterminer la saison de végétation, nous avons choisi la température-seuil de $7{ }^{\circ} \mathrm{C}$ proposée par PARDÉ (1959) et nous avons calculé la liaison entre les dates ( $1^{\text {er }}$ au $365^{\circ}$ jour) du début et de la fin de la saison de végétation, ainsi que sa durée à partir des températures moyennes mensuelles de la région, après interpolation linéaire. Les liaisons sont linéaires et il existe évidemment une très bonne corrélation avec l'altitude.

$$
\begin{array}{lll}
Y=42,57+6,63 X & R=0,996 & s y=3,04 \\
Y^{\prime}=335,38-3,79 X & R=0,981 & s y^{\prime}=3,84 \\
Y^{\prime \prime}=292,81-10,42 X & R=0,993 & s y^{\prime \prime}=6,33
\end{array}
$$

où :

$Y$ est le ième jour de l'année du début de la période de végétation

$Y^{\prime}$ est le ième jour de l'année de la fin de la période de végétation

$Y^{\prime \prime}$ est la durée de la période de végétation en jours

$\mathrm{X}$ est l'altitude exprimée en mètre et multipliée par $10^{-2}$.

La figure 3, qui représente ces trois droites de régressions, permet certaines constatations : la période de végétation commence 6,6 jours plus tard pour une élévation de $100 \mathrm{~m}$ en altitude; tandis que la fin de la période de végétation change moins rapidement ( 3,8 jours pour $100 \mathrm{~m}$ de dénivellation) ceci provoque une certaine asymétrie des saisons. La durée diminue de 10,4 jours pour $100 \mathrm{~m}$ de dénivellation.

A $1600 \mathrm{~m}$ d'altitude, le seuil de $7{ }^{\circ} \mathrm{C}$ est atteint vers le 29 mai, la température reste supérieure à $7{ }^{\circ} \mathrm{C}$ pendant environ 126 jours et tombe en dessous du seuil à partir du 2 octobre.

\subsection{4. - Le vent}

Parmi les facteurs du climat qui agissent sur la production forestière, c'est le vent qui, dans cette région, a un rôle prépondérant. Son action physiologique et mécanique sur les arbres est, dans son effet global, bien connue (PERRIN, 1963). 


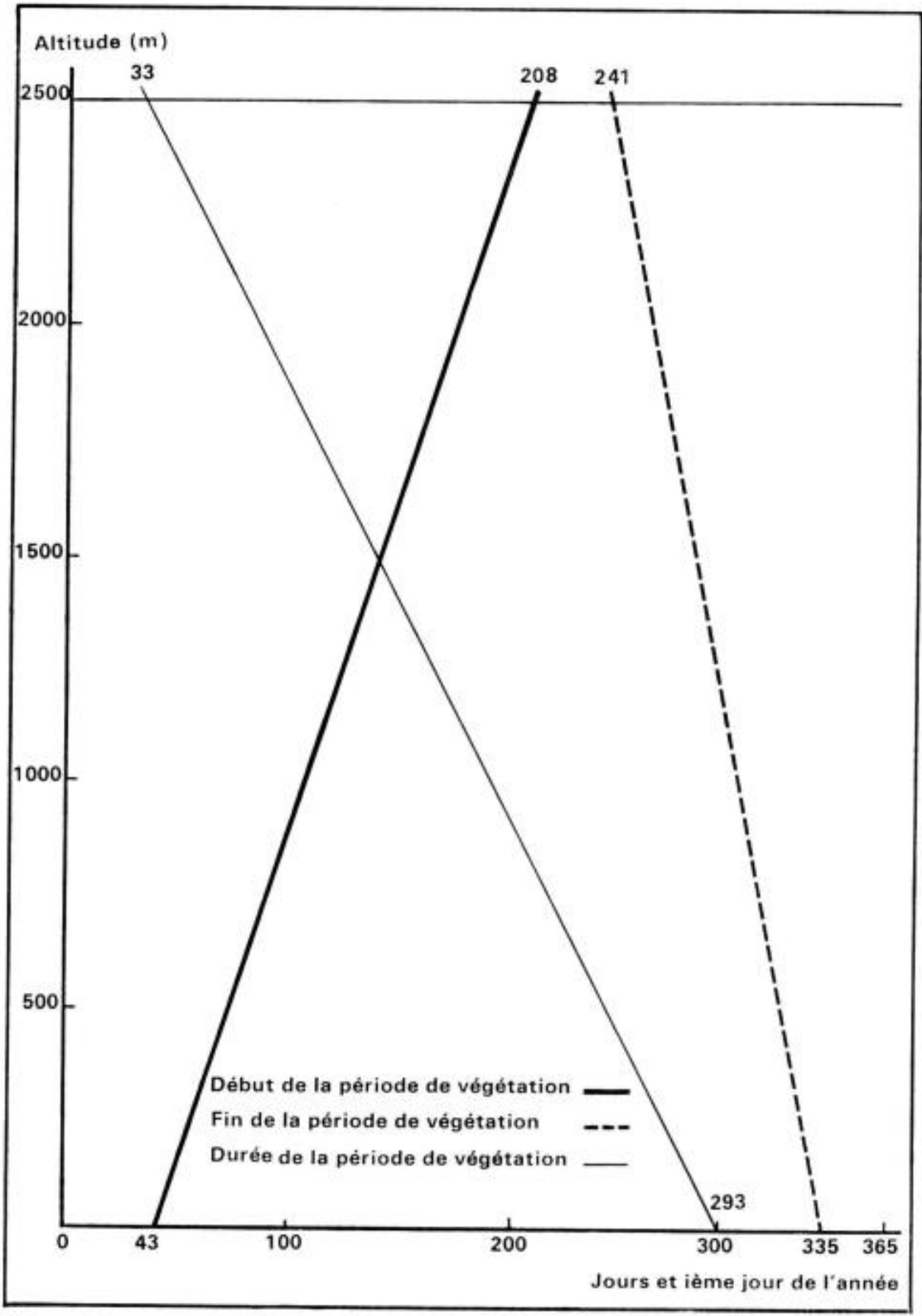

Fig. 3. - Début, fin et durée de la période de végétation $\left(\mathrm{t}>7^{\circ} \mathrm{C}\right)$ en fonction de laltitude 
Le vent est le facteur déterminant de la limite supérieure de la forêt dans le Massif Central. D'après P. EsTIENNE (1956) les principaux vents sont le vent du Nord, le plus fréquent, responsable de tempêtes de neige et des congères en hiver, et le vent du Sud, le plus violent et qui apporte souvent des pluies abondantes. Les calmes sont rares (environ $3 \%$ au Mont-Aigoual), les variations saisonnières faibles : la vitesse du vent est relativement élevée et elle augmente avec l'altitude. Les vents du secteur Est sont pratiquement sans importance. Ce même auteur souligne également l'effet prépondérant du relief local sur la direction et la vitesse du vent. En fait, nous ne possédons pas de données quantitatives sur le vent dans notre zone d'action, mais nous avons cru bon de tenir compte de ce facteur dans la description de nos peuplements.

Dans ce but, nous avons calculé les fréquences moyennes des directions du vent, calmes exclus, de trois stations qui entourent notre région, Privas, Le Puy et le Mt-Aigoual (tab. 8).

\section{TABLEAU 8}

Fréquences pour 1000 des directions du vent (calmes exclus) d'après IOO.N.M. (1942 a, 1942 b)

\begin{tabular}{|c|c|c|c|c|c|c|c|c|c|}
\hline Station & $\mathrm{N}$ & $\mathrm{NE}$ & $\mathrm{E}$ & SE & S & SW & w & NW & Total \\
\hline Le Puy ................... & 197 & 131 & 49 & 59 & 171 & 113 & 133 & 147 & 1000 \\
\hline Privas ................... & 470 & 39 & 20 & 37 & 345 & 29 & 23 & 37 & 1000 \\
\hline Mont Aigoual ............ & 278 & 35 & 16 & 65 & 221 & 64 & 68 & 253 & 1000 \\
\hline Moyenne ... & 315 & 68 & 28 & 54 & 246 & 68 & 75 & 146 & 1000 \\
\hline
\end{tabular}

En interpolant linéairement entre les 8 directions principales, nous avons déterminé une fréquence moyenne de 10 en 10 grades pour un secteur de 50 grades. Les valeurs ainsi déterminées furent groupées en $5+1$ classes $(0$ à 6$)$. La valeur zéro était attribuée aux stations abritées et la valeur six aux stations exposées à tous les vents, et aux stations où les dégâts du vent (cimes en drapeaux, etc.) étaient nettement apparents. Cette échelle de notations n'est exacte que pour ses valeurs extrêmes, mais elle a l'avantage d'être objective et qualitative et reproduit un effet combiné de la fréquence du vent et de l'exposition. Pour certains calculs statistiques, nous avons appliqué une transformation en arc sin $\sqrt{\frac{x}{6}(\%)}$ $(\mathrm{x}=$ classes de 1 à 6 ) d'après des Tables de SNEDECOR (1962).

Dans ce classement, nous n'avons évidemment pas tenu compte de la vitesse du vent, qui augmente avec l'altitude, et nous avons certainement sous-estimé le vent du Sud. Pour combler ces lacunes, il faudrait faire une étude régionale du vent. 


\subsection{5. - L'évapotranspiration potentielle}

L'évapotranspiration potentielle (ETP) est un élément synthétique très important du climat. Suivant la formule proposée par TuRC (1961) ('), nous avons calculé l'ETP mensuelle et annuelle pour 9 stations à différentes altitudes. Dans nos calculs de bilans hydriques, nous avons appliqué une valeur de $100 \mathrm{~mm}$ à la réserve d'eau utile ou facilement évaporable dans le sol (RFU), mais nous n'avons pu tenir compte ni de l'influence du relief, ni de celles de l'exposition et de la pente; nous avons également négligé l'interception de la couverture végétale ainsi que l'influence des pluies à forte intensité sur l'écoulement des précipitations en surface. L'ETP diminue comme la température, linéairement avec l'altitude et nous avons trouvé une diminution de l'ETP annuelle d'environ $27 \mathrm{~mm}$ pour une élévation de $100 \mathrm{~m}$ dans la région étudiée.

Les principaux résultats peuvent être résumés ainsi :

- l'ETP mensuelle est supérieure aux précipitations pendant 5 mois (mai à septembre) au Puy et pendant le mois de juillet au Mt-Mézenc (M.F.).

- le déficit total en eau (RFU $=100 \mathrm{~mm}$ ) est de :

$86 \mathrm{~mm}$ (juillet et août) à Aubenas,

$118 \mathrm{~mm}$ (juillet à septembre) au Puy,

$27 \mathrm{~mm}$ (août) à Langogne,

$0 \mathrm{~mm}$ à Issanlas,

$8 \mathrm{~mm}$ (en août) à Sainte-Eulalie (M.F.).

$0 \mathrm{~mm}$ au Mont-Mézenc (M.F.).

- l'écoulement théorique est de $54 \mathrm{~mm}(8 \%)$ au Puy et de $813 \mathrm{~mm}(64 \%)$ au Mont-Mézenc (M.F.).

Ces résultats montrent qu'au-dessus de $1000 \mathrm{~m}$ d'altitude, les déficits estivaux en eau sont rares et peu importants, mais qu'ils peuvent cependant se produire pendant les années sèches.

\subsection{6. - Evapotranspiration potentielle el production}

De nombreux auteurs ont essayé de mettre au point des indices de produccivité à partir des données climatiques. Ainsi PATTERSon (1956) a établi l'indice CVP et PARDÉ (1964) l'applique à la France après modification. RoBLIN (1961) a pu montrer qu'il existe, dans une certaine limite, une liaison linéaire entre le rendement en matière sèche et l'évapotranspiration réelle (ETR) ; tout récemment L. TuRC (1967) a publié une intéressante étude sur un indice climatique de potentialité agricole qui permet de relier la production annuelle en matière sèche, spécialement celles des plantes fourragères, à des données macroclimatiques. Une

(1) Dans la formule de Turc, nous avons utilisé la đurée d'insolation du Puy pour l'ensemble de 9 stations. L'erreur ainst introduite est inferieure a $10 \%$. 
application aux hautes terres du Massif Central, entre 700 et $1500 \mathrm{~m}$, met en évidence une situation assez favorable en ce qui concerne la production fourragère ; l'indice prend des valeurs de 20 à 25 , tandis que dans la plus grande partie de la France, cet indice oscille entre 20 et 30 . Il faut également mentionner ici le \& coefficient bioclimatique de productivité potentielle » de GiacoBbe (1967) comme l'un des 3 facteurs de la productivité, les deux autres étant le sol et la plante. Nous n'avons pas essayé d'établir une liaison d'un tel indice ou coefficient avec la production forestière, car faute de données stationnelles, ils ne peuvent avoir de signification que pour l'ensemble d'une région.

\subsection{7. - Conclusion sur le climat}

La Haute-Ardèche, au climat montagnard, est située au carrefour de trois influences climatiques, méditerranéenne, continentale et atlantique, et est caractérisée par une très grande variabilité de tous les éléments qui constituent le climat, variabilité dans le temps et dans l'espace.

Si pour l'ensemble de la région les précipitations sont relativement élevées au-dessus de $1000 \mathrm{~m}$ d'altitude, des déficits en eau peuvent se produire certaines années pendant l'été, même au-dessus de $1300 \mathrm{~m}$ d'altitude et surtout dans la partie sud de la région, où il existe, en juillet, un minimum prononcé des précipitations ; en plus, il faut tenir compte de l'énorme intensité des pluies, de l'écoulement superficiel et de l'interception par la végétation. P. EsTIENNE (1956) a souligné la grande variabilité du climat du Massif Central ; ceci est particulièrement vrai pour toutes les formes de précipitations et elle est sensiblement plus grande dans les zones à influence méditerranéenne.

\section{5. - La végétation}

\subsection{1. - Les étages de végétations}

De nombreuses études ont été faites sur la zonation altitudinale de la végétation dans le Massif Central (L. Beille, 1889 ; J. Carles, 1956). A l'exception de quelques stations sommitales où l'on peut parler d'un étage pseudo-alpin (FLAHaUlT, 1901), la région étudiée se trouve entièrement dans l'étage montagnard du Sapin et du Hêtre, dont la limite inférieure se situe entre 800 et $1000 \mathrm{~m}$ d'altitude (J. CARLES, 1953).

\subsection{2. - Description des forêts actuelles}

Nous savons aujourd'hui par de nombreuses analyses polliniques (LEMEE G., 1953-1956) que le déboisement de cette région est très ancien, antérieur à tous les documents historiques et nous connaissons l'origine ainsi que l'évolution de nos essences forestières (Guinier Ph., 1956 ; LEMEE G., 1953-1956). 


\subsection{Le Pin sylvestre (Pinus sylvestris $L$. )}

Quoique spontané dans la région (* le Pin d'Auvergne \$), la plupart des peuplements de cette essence sont d'origine artificielle. L'importance du pin dans le Massif Central est considérable surtout en basse et moyenne altitude (300 à $1200 \mathrm{~m}$ ) et selon CARLEs (1947), on peut même parler d'un étage du Pin sylvestre. Cette essence convenait paraitement aux exigences des agriculteurs par son installation facile et par la possibilité de pacage sous son couvert léger. Le Pin sylvestre joue sensiblement le même rôle dans l'économie rurale du Massit Central que le mélèze dans les Alpes sèches.

La majorité des peuplements appartient à des propriétés privées, mais il s'agit exclusivement de petites propriétés très morcelées, à économie mixte. Les forêts de pins sont issues de plantations après cultures agricoles ou après coupe à blanc. Elles sont traitées en futaie régulière à courte révolution (30 à 60 ans) et produisent essentiellement des bois de mines et des poteaux. Depuis une dizaine d'années, ce marché est en constante dégradation et les débouchés pour le bois de Pin sylvestre de faible dimension deviennent de plus en plus difficiles à trouver. Pour être complet, il est curieux de mentionner la production locale de bois de boulange en fagots, " la garne ", par émondage (GalloIs, 1933). Ce mode particulier d'exploitation est à l'abandon mais il y a encore quelques peuplements, * les garnasses , qui en témoignent.

Le Pin sylvestre peut être considéré comme une essence pionnière et au fur et à mesure que l'agriculture abandonne ou sous-exploite certains terrains, il s'y installe naturellement. L'état des peuplements est souvent très médiocre, l'intervention sylvicole est quasi absente et le pacage est partout de rigueur.

\subsection{Le Hêtre (Fagus silvatica L.)}

Le Hêtre qui occupe les ubacs entre 800 et $1200 \mathrm{~m}$ et monte aux adrets jusqu'à environ $1450 \mathrm{~m}$ d'altitude, ne constitue que quelques rares futaies pures ; il est souvent mélangé avec le Sapin pectiné dans des proportions variables, et s'il est en peuplement pur, traité en taillis simple. Son bois est d'une qualité très médiocre et son importance économique très faible. Les taillis de Hêtre d'altitude sont de moins en moins exploités et leur enrésinement s'impose.

\subsection{Le Sapin pectiné (Abies alba Mill.)}

Le Sapin pectiné est largement représenté surtout dans toutes les forêts domaniales (Mazan, Bonneroy, Lac du Bouchet, etc.), mélangé en proportions variables avec le Hêtre : il occupe tout l'étage montagnard et peut atteindre des dimensions respectables. Les peuplements sont traités en futaie jardinée, mais il existe souvent un excédent de vieux bois et il semble difficile d'approcher la structure idéale de la futaie jardinée.

La régénération naturelle peut être acquise assez facilement sur des stations fraîches, mais la dominance du Hêtre sur les stations plus sèches nécessite l'intervention constante du sylviculteur en faveur du Sapin pour maintenir un certain équilibre entre ces deux essences. 
Le bois du sapin est de bonne qualité et utilisé surtout en sciages.

\subsection{Le Pin à crochets (Pinus uncinata Ram.)}

Une des particularités de la flore forestière du Massif Central est la rare présence spontanée du $\mathrm{P}$ in à crochets dans quelques tourbières. Mais cette essence fut également utilisée dans des reboisement en altitude, à la fin du siècle dernier, notamment au Mont-Mézenc, en Forêt domaniale de Bonnefoy et dans quelques séries RTM sur des stations sommitales. A l'âge de 80 ans, ces peuplements sont dépérissants et leur production extrêmement faible, mais on rencontre souvent une belle régénération naturelle de Sapin pectiné sous leur couvert léger.

\subsection{L'Épicéa commun (Picea Abies L.)}

Il n'est pas besoin de souligner l'importance de cette essence pour les reboisements anciens et futurs dans le Massif Central, notamment aux altitudes supérieures à $1000 \mathrm{~m}$, où elle constitue déjà d'importants massifs. Les peuplements sont à l'état pur et équienne mais souvent aménagés en futaie jardinée.

Pourtant, selon de nombreux auteurs et notamment Ph. GuinIER (1956) l'Épicéa commun n'est pas spontaané dans le Massif Central et son introduction a commencé au début du $19^{\circ}$ siècle. On peut dire aujourd'hui que l'Épicéa est définitivement installé dans le Massif Central et il y a toute chance que son importance s'accentue considérablement dans les années à venir.

\subsection{Autres essences de reboisement}

Un grand nombre d'essences, telles que Pin cembro, Mélèze d’Europe, Pin noir d'Autriche et autres, furent essayées dans les premiers travaux de reboisement RTM à la fin du siècle dernier, mais ces tentatives se soldèrent souvent par un échec total pour des raisons diverses (BUFFAULT, 1916 ; GUINIER, 1952).

\subsection{3. - Les groupements végétaux}

Dans chacune de nos placettes, nous avons effectué des relevés phytosociologiques avec le concours de G. Aussenac qui a également étudié 30 relevés dans la lande. Nous ne voulons pas présenter ici les résultats d'une étude phytosociologique que nous publierons à une date ultérieure. L'exploitation de nos relevés et de nos observations sur le terrain, en ce qui concerne la répartition de différents types de landes, nous permet de constater qu'ils sont sensiblement les mêmes que ceux décrits par B. Peyre de Fabregues (1962) dans le Massif de l'Aigoual et ceux de P. DAget et J. Poissonet (1966) dans la Margeride.

Nous retenons deux types de lande floristiquement et physionomiquement très différents :

- Lande à Callune avec plusieurs faciès (Cailuna vulgaris, Nardus stricta. Vaccinium Myrtillus, etc.): 
- Lande à genêts : Genista purgans, Sarothamnus scoparius et Pteridium aquillinum ; ces trois espèces constituent trois faciès distincts suivant leur dominance.

Ces formations de lande, d'origine anthropo-zoogène (déboisement, écobuage, pacage, etc.) évoluent très lentement. Aucune n'oppose d'obstacle sérieux au reboisement.

Les groupements végétaux rencontrés en forêts artificielles et naturelles correspondent sensiblement aux groupements décrits par Ch. BARTOLI (1961) dans le procès-verbal de Révision d'Aménagement (1961-1986) $1^{\text {re }}$ série de la forêt domaniale de Mazan, par G. LemeE et R. CArbinier (1956) dans la chaine des Puys, et par Ph. Daget et J. Poissonet (1966) dans la Margeride qui ont également étudié les pelouses et les prairies artificielles.

\section{II. - METHODES ET MATERIEL UTILISES}

\section{1. - Généralités}

Une étude de potentialités forestières consiste essentiellement à tenter d'établir une liaison production-station. La notion de \& production potentielle $» n$ 'indique finalement que la production actuelle dans des conditions de milieu existant.

La présence d'un nombre suffisant de peuplements d'épicéa en Haute-Ardèche et en Haute-Loire, a orienté notre choix vers cette essence, d'autant plus qu'elle constitue, avec le Sapin, la seule essence de reboisement commercialement rentable et utilisable dans les hautes terres de cette région.

Une table de production régionale constitue évidemment la meilleure base d'une étude de potentialités. Elle est un modèle mathématique et tient compte d'un grand nombre de paramètres, tels la * fertilité * et les \& traitements sylvicoles *. Malheureusement, il n'en existe pas encore pour l'Épicéa.

L'absence totale de dispositifs expérimentaux permanents dans la région et limpossibilité d'estimer la production totale ou laccroissement à partir des procès-verbaux d'aménagement, nous ont obligés à installer des placettes temporaires ou semi-permanentes (PARDE J. 1966).

Il est généralement admis qu'une série de placettes temporaires dâages différents sur des stations comparables, peut être confondue avec l'évolution d'un peuplement dans le temps. Magin (1967) a élaboré une formule permettant une bonne estimation de la production totale à partir d'une telle série. Mais il nous était impossible d'utiliser cette formule car la plupart des peuplements de la région ont sensiblement le même âge ( 76 ans : âge moyen des 33 peuplements).

Pour pallier cet inconvénient, nous avons eu recours à l'analyse de tiges d'arbres dominants pour reconstituer de façon valable lévolution dans le temps de tel ou tel peuplement. Cette méthode est largement employée et sa validité ne fait plus de doute (CURTIS R.O., 1964 ; MiNor Ch.O., 1964 ; AssmanN E., 1967).

\section{2. - Variables des peuplements}

En ce qui concerne les mesures effectuées dans les placettes, nous avons strictement suivi les instructions de la note interne de N. DecourT (1966) dans le souci d'utiliser ces placettes ultérieurement pour l'établissement d'une table de production.

En plus de ces mesures habituelles (1), nous avons choisi deux arbres de l'étage dominant sans défaut apparent et dont les dimensions correspondaient aux valeurs moyennes calculées

(1) On trouvera dans la légende du tableau 9, la définition des symboles dendrométriques que nous avons čtế amenés à employer. 


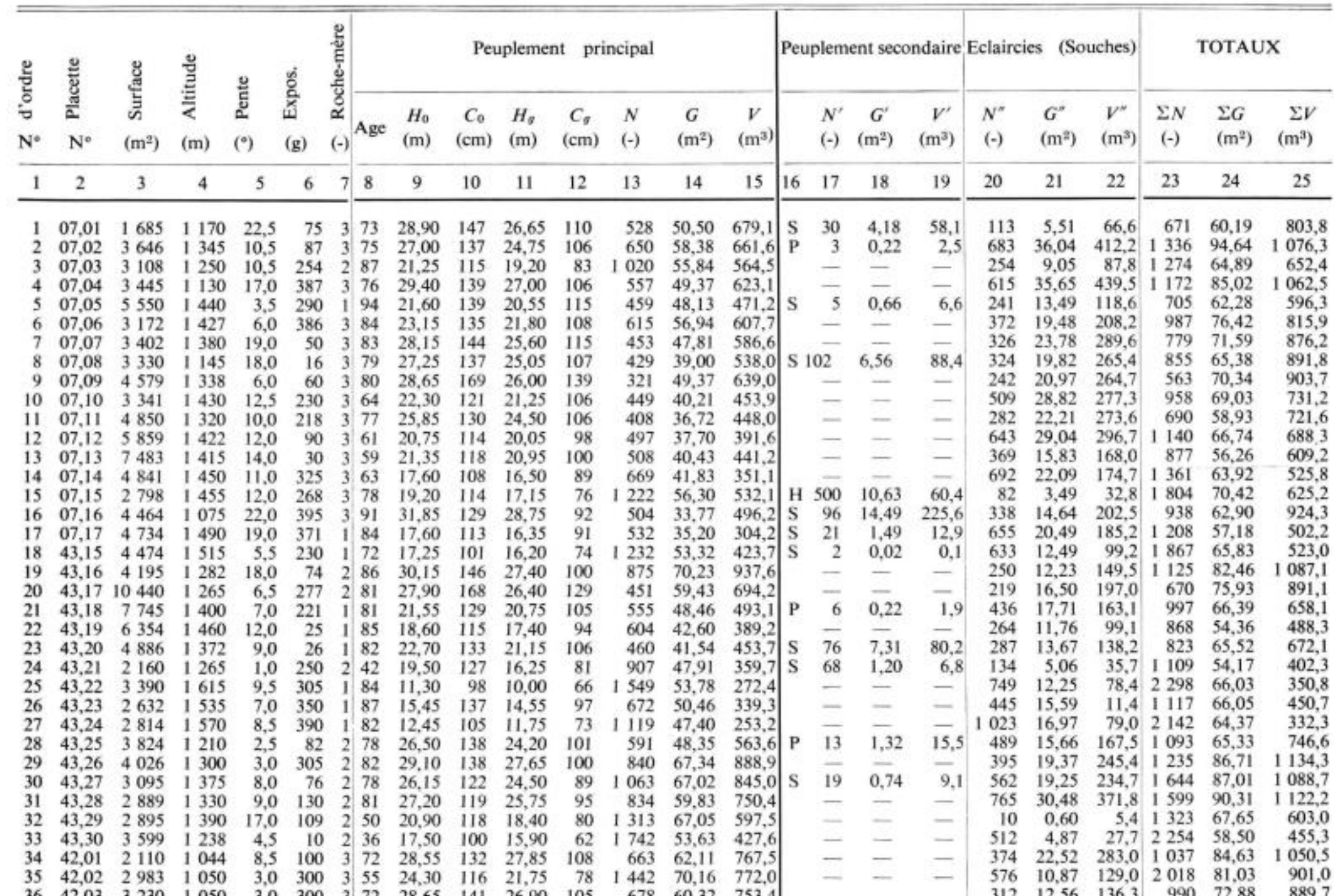




\begin{tabular}{|c|c|c|c|c|c|c|c|c|c|c|c|c|c|c|c|c|c|c|c|c|c|c|c|c|}
\hline 4. & $\begin{array}{l}43,14 \\
43,14^{*}\end{array}$ & $\begin{array}{l}3736 \\
4150\end{array}$ & $\begin{array}{l}1280 \\
1360\end{array}$ & 8,0 & 225 & & 85 & 80 & 137 & 3,30 & 11 & 548 & 54,21 & 567,2 & & - & - & - & 513 & 19,33 & 219,6 & 1061 & 73,54 & 786,8 \\
\hline 42 & $43, \mathrm{Al}$ & 400 & 1285 & 1,5 & 260 & 3 & 19 & $\begin{array}{r}23,43 \\
7,01\end{array}$ & $\begin{array}{r}134 \\
37\end{array}$ & $\begin{array}{r}22,50 \\
500\end{array}$ & $\begin{array}{r}112 \\
28\end{array}$ & $\begin{array}{r}412 \\
2725\end{array}$ & $\begin{array}{l}41,35 \\
1173\end{array}$ & 474,4 & & $\overline{203}$ & - & - & 546 & 23,29 & 246,5 & 958 & 64,64 & 720,9 \\
\hline 43 & $43, \mathrm{~A} 2$ & 400 & 1280 & 4.5 & 230 & 3 & 19 & 5,81 & 29 & $\begin{array}{l}4,00 \\
4,08\end{array}$ & $\begin{array}{l}28 \\
18\end{array}$ & 2750 & $\begin{array}{r}11,73 \\
7,06\end{array}$ & - & $\mathrm{P}$ & 225 & E & - & - & I & - & 二 & - & - \\
\hline & $43, \mathrm{~A} 3$ & 400 & 1258 & 3,0 & 330 & 3 & 19 & 5,65 & 28 & 3.65 & 15 & 3625 & 6,27 & - & $\mathrm{P}$ & 500 & - & - & - & - & - & 二 & E & - \\
\hline 45 & $43, \mathrm{~A} 4$ & 400 & 1268 & 5,0 & 320 & 31 & 19 & 6,20 & 31 & 4,65 & 22 & 2750 & 9,94 & - & & - & - & - & - & - & - & Z & E & - \\
\hline & 43,BI & 400 & 1245 & 5,5 & 295 & 31 & 13 & 3,33 & 14 & 2,28 & 8 & 2875 & 1,35 & - & & - & - & - & - & - & - & - & - & - \\
\hline 47 & $43, B 2$ & 400 & 1238 & 5,5 & 350 & 31 & 13 & 2,88 & 12 & 2,15 & 7 & 2675 & 1,00 & - & & - & - & - & - & - & - & - & - & - \\
\hline & $43, \mathrm{Cl}$ & 400 & 1207 & 4.5 & 300 & 21 & 15 & 5,25 & 26 & 3,84 & 17 & 2400 & 4,95 & - & P & 425 & - & - & - & - & - & - & - & - \\
\hline 49 & $43, \mathrm{C} 2$ & 400 & 1217 & 2,5 & 300 & 21 & 15 & 5,30 & 27 & 3,98 & 18 & 2550 & 6,23 & - & $\mathrm{P}$ & 50 & - & - & - & - & - & - & - & - \\
\hline 50 & $07, \mathrm{Al}$ & 400 & 1278 & 8,5 & 54 & 2 & 8 & 0,84 & - & - & - & 3475 & - & - & & - & - & - & - & - & - & - & - & - \\
\hline 51 & $07, \mathrm{~A} 2$ & 400 & 1280 & 8,5 & 54 & 2 & 8 & 0,79 & - & - & - & 3350 & - & - & & - & - & - & 一 & - & - & - & - & - \\
\hline 52 & $07, \mathrm{~A} 3$ & 400 & 1320 & 10,0 & 40 & 2 & 8 & 0,62 & - & - & - & 2400 & - & - & P & 25 & - & - & - & - & - & - & - & - \\
\hline 23 & $07, \mathrm{~A} 4$ & 400 & 1320 & 10,0 & 40 & 2 & 8 & 0,85 & - & - & - & 2400 & - & - & P & 50 & - & - & - & - & - & - & - & - \\
\hline 54 & $07, \mathrm{~A} 5$ & 400 & 1310 & 9,5 & 45 & 2 & 8 & 0,67 & - & - & - & 2875 & - & - & & - & - & - & - & - & - & - & - & - \\
\hline 55 & $07, \mathrm{~A} 6$ & 400 & 1295 & 9,5 & 45 & 2 & 8 & 0,85 & - & - & - & 3125 & - & - & & - & - & - & - & - & - & - & - & - \\
\hline 56 & 07,01 & 3443 & 1145 & 3,0 & 235 & 3 & 90 & 18,75 & 112 & 17,35 & 87 & 706 & 42,99 & 325,5 & & - & - & - & 331 & 10,32 & 75,2 & 037 & 53,31 & 400,6 \\
\hline 58 & 43,01 & $\begin{array}{l}3309 \\
364\end{array}$ & 1090 & 19,0 & 103 & 1 & 150 & 20,70 & 131 & 17,90 & 97 & 795 & 59,19 & 523,6 & & - & - & 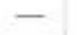 & 10 & 4,1 & 30,9 & 901 & 63,37 & 554 \\
\hline 58 & 43,02 & 2665 & 840 & 15,0 & 87 & ? & 76 & 15,70 & 93 & 14,05 & 57 & 1666 & 43,26 & 291,6 & & - & - & - & 86 & 0,43 & 1,3 & 1752 & 43,69 & 293,0 \\
\hline 59 & 43,01 & 3115 & 1098 & 1,0 & 80 & $2 \theta$ & 61 & 21,00 & 121 & 19,40 & 86 & 1172 & 69,02 & 667,9 & & - & - & - & 411 & 13,77 & 131,9 & 1583 & 82,79 & 799,9 \\
\hline 60 & 0,701 & 1780 & 1010 & 25,0 & 80 & 3 & 67 & 27.40 & 156 & 25,60 & 110 & 663 & 63,48 & 688,9 & P & 140 & 7,34 & 55,0 & 348 & 5,26 & 42,7 & 1151 & 76,08 & 786,6 \\
\hline
\end{tabular}

Colonne 1: $\mathrm{N}^{*}$ d'ordre de la placette

1 à 33 : placettes temporaires d'Épicéa installées en 1966 et 1967 34 à 41 : placettes temporaires d'Épicéa installées en 1961. 42 à 55 : placettes dans des plantations d'Épicéa installéesen 1967. 56 à 58 : placettes temporaires de Pin sylvestre installées en 1967. 59 : placette temporaire de Sapin pectiné installée en 1967. 60 : placette temporaire de Douglas installée en 1967

Colonne $2: \mathrm{N}^{\circ}$ de la placette ; les deux premiers chiffres indiquent le $\mathrm{n}^{\circ} \mathrm{du}$ département $(07=$ Ardèche $)(42=$ Loire $)(43=$ Haute-Loire $)$.

Colonne 3 : surface inventoriée de la placette en $\mathrm{m}^{2}$.

Colonne 4 : altitude en métres.

Colonne 5: pente en degrés.

Colonne 6 ; exposition en grades.

Colonne 7 : roche-mère $\left(1=\right.$ phonolite, $2=$ basalte, $3=$ granite, ${ }^{*}=$ argiles et sables à chailles).

Colonnes 8 à 15 : peuplement principal (Êpicéa).

Colonne $8: A$; âge moyen (années).

Colonne 9: $H_{0}$; hauteur dominante $\left(H_{0}\right)$ (hauteur de l'arbre de surface terrière moyenne des 100 plus gros arbres à l'hectare) en mètres.

Colonne 10: $C_{0}$; circonférence dominante à $1,30 \mathrm{~m}$ ( = circonférence de l'arbre de surface terrière moyenne des 100 plus gros arbres à I'hectare) en $\mathrm{cm}$.
Colonne $11: H_{0}$; hauteur moyenne (= hauteur de l'arbre de surface terrière moyenne en $m$ ).

Colonne $12: C_{g}$; circonférence moyenne à $1,30 \mathrm{~m}$ (= circonférence de larbre de surface terrière moyenne) en $\mathrm{cm}$.

Colonne $13: N$; nombre de tiges à I'hectare.

Colonne $14: G ;$ surface terriere en $\mathrm{m}^{2} / \mathrm{ha}$.

Colonne 15: $V$; volume sur pied du peuplement ; bois-fort tige sur écorce en $\mathrm{m}^{3} /$ ha.

Colonnes 16 à 19 : peuplement secondaire (en mélange).

Colonne 16 ; essence en mélange

$$
\mathrm{H} \text { : Hêtre. } \mathrm{P} \text { : Pin sylvestre. } \mathrm{S} \text { : Sapin pectiné. }
$$

Colonne $17: N^{\prime} ;$ nombre de tiges à l'hectare.

Colonne $18: G^{\prime} ;$ surface terrière $\left(\mathrm{m}^{2} / \mathrm{ha}\right)$.

Colonne 19: $V^{\prime} ;$ volume sur pied $\left(\mathrm{m}^{3} / \mathrm{ha}\right)$.

Colonnes 20 à 22 : éclaircies récentes d'après les souches.

Colonne $20: N^{\prime \prime} ;$ nombre de souches à l'hectare.

Colonne 21: $G^{\prime \prime} ;$ surface terrière $\left(\mathrm{m}^{2} / \mathrm{ha}\right)$.

Colonne $22: V^{\prime \prime} ;$ volume $\left(\mathrm{m}^{3} / \mathrm{ha}\right)$ d'aprés les souches.

Colonnes 23 à 25 : Totaux à l'hectare.

Colonne $23: N ; N+N^{\prime}+N^{\prime}$

Colonne $24 ; G ; G+G^{\prime}+G^{\sigma}$.

Colonne $25: V ; V+V^{\prime}+V^{n}$. 
de la placette (circonférence dominante et hauteur dominante du peuplement principal).

- hauteur totale,

- hauteur bois fort,

- circonférence à la souche,

- circonférence à $1,30 \mathrm{~m}$ du sol,

- circonférence aux 9 niveaux relatifs (1),

- âge de la souche,

- âge à $1,30 \mathrm{~m}$ du sol,

- âge aux 9 niveaux relatifs,

- rayon moyen d'une rondelle découpée à $1,30 \mathrm{~m}$ d'année en année selon la méthode préconisée par R. SIOSTRZONEK (1958).

Dans chaque placette, nous avons également mesuré les quatre plus gros arbres et prélevé une carotte à $1,30 \mathrm{~m}$ du sol dans une direction choisie au hasard pour déterminer leurs âges à $1,30 \mathrm{~m}$ et l'infra-densité moyenne des cernes pour les 30 dernières années.

Chaque peuplement faisait l'objet d'une inscription détaillée (état sanitaire, élagage, régénération, traitement sylvicole, estimation des produits, etc.). Nous avons également inventorié un certain nombre de jeunes plantations.

\section{2,3. - Variables du milieu}

Dans tous les peuplements, nous avons effectué une description détaillée de la station (relevé phytosociologique, description et analyse physique et chimique du sol à partir d'une fosse pédologique profonde, etc,). Mais nous n'avons retenu, dans un premier stade, que des variables qui sont facilement mesurables ou repérables sur le terrain, et qui peuvent en partic être déterminées sur des cartes topographiques et géologiques à grande échelle $(1 / 25000)$. comme l'altitude, l'exposition, la pente, la nature de la roche mère, etc.

\section{4. - Méthodes et moyens de calcul}

L'ensemble des données, dont la partie essentielle est présentée dans le tableau 9, fut stocké sur disque et traité sur ordinateur IBM $1130(8 \mathrm{~K})$ sous la direction de R. Tomassonł de la Station de Biométric, Un certain nombre de calculs préliminaires ou mineurs furent exécutés sur Olivetti-Programma 101.

Comme méthodes de calcul, nous avons essentiellement employé l'analyse de régression (régression polynomiale, régression multiple linéaire, régression progressive multiple ${ }^{2}$ )) qui permet d'expliquer une variable $Y$ (variable expliquée) en fonction de $p$ variables explicatives $X_{1}, X_{2} \ldots X_{p}$. L'ajustement se fait sur un modèle linéaire par la méthode des moindres carrés (R. TOMASSONE 1967 a - 1967 b).

Les différents programmes de la Station de Biométrie permettent en outre de nombreuses transformations des variables en vue d'obtenir le meilleur ajustement. Nous nous bornerons à indiquer les seuls résultats retenus soit pour leur précision suffisante, soit pour leur simplicité relative.

\section{III. - RESULTATS ET DISCUSSION}

\section{1. - RElations entre L.es VARIABles dU PEUPlement ET LES FACTEURS DU MILIEU}

\subsection{1. - Relation hauteur-âge}

Dans un premier stade, nous avons vérifier que la hauteur des arbres choisis pour l'analyse de tige n'était pas significativement différente de la hauteur dominante du peuplement correspondant.

(1) Découpe de la tige en 10 sections de longueur relative égale (1/10 de la hauteur totale).

(2) Dans la régression progressive multiple, les variables explicatives sont introduites une à une dans un ordre tel, qu'elles fournissent la meilleure réduction de la somme de carrés résiduelle. L'introduction pas à pas (résultats intermédiaires) permet également de mieux analyser les relations entre les variables explicatives et de connaître lapport propre à chaque nouvelle variable, associée à celles déjà introduites. 
Pour chacun de ces arbres, nous avons ajusté la relation hauteur-âge (11 niveaux par arbre) par un polynôme du $3^{r}$ degré. L'équation est de la forme :

$$
\begin{aligned}
& Y=a_{0}+a_{1} X+a_{2} X^{2}+a_{3} X^{3} \\
& Y=\text { hauteur à un niveau donné, } \\
& X=\text { âge à un niveau donné. }
\end{aligned}
$$

La régression polynomiale était meilleure qu'un ajustement après transformation logarithmique et nous avons obtenu un coefficient de corréla ion mul.iple $\left(R^{2}\right)$ de l'ordre de 0,98 avec un écart type résiduel $(s \bar{y})$ qui est en moyenne de $66 \mathrm{~cm}$.

A partir de cette équation, nous avons calculé successivement les hauteurs correspondantes aux âges 25,50 et 75 ans et nous rappelons $\bar{h} o$ (25), $\bar{h} o$ (50) et ho (75) les valeurs moyennes des deux arbres d'une placette.

\subsection{2. - Relations hauteur dominante-milieu}

Dans une première série de régressions progressives multiples, nous avons expliqué la hauteur dominante réelle du peuplement $(\mathrm{Ho})$ et la hauteur moyenne des deux arbres analysés ( $\bar{h} o$ ) dans ce même peuplement, en fonction des variables stationnelles telles que l'altitude, la pente, lexposition, la position topographique (1), la roche mère et le vent, sans tenir compte de l'âge, et nous pouvons faire les observations suivantes :

- l'introduction des variables se fait toujours dans le même ordre d'importance décroissante : altitude, vent, position topographique (ces 3 variables sont significatives), Ho et ho,

- les coefficients de ces 3 variables sont sensiblement les mêmes pour

- la pente, l'exposition et le substratum ne sont pas significatifs,

(1) La position topographique fut notée à l'aide d'un code numérique; dix positions différentes ont étét retenues :

0 : Sommet arrondi et croupe.

1: Arête ou ligne de crête.

2: Haut de versant et bord de plateau.

3: Mi-versant.

4: Bas de versant.

5 : Terrain plat.

6: Falaise.

7: Cuvette fermée.

8 : Fond de vallée et dépression.

9: Terrasse et gradin.

Ce code a l'inconvénient d'ètre discontinu et qualitatif. Dans certains cas, la position topographique peut être exprimée d'une manière quantitative et continue, mais comme la majorité de nos placettes est située sur mi-versant et quelques placettes seulement sur haut de versant, bas de versant et terrain plat, nous n'avons pas retenu cette possibilité. 
- pour les 3 variables significatives, les coefficients de corrélation multiple et les écarts-types résiduels ne sont pas significativement différents ; on obtient pour

$$
\begin{aligned}
& \text { Ho: } R^{2}=0,776, s=2,634 \mathrm{~m} \\
& \bar{h} o: R^{2}=0,719, s=2,930 \mathrm{~m} .
\end{aligned}
$$

Si par contre, on tient compte de l'âge, c'est-à-dire, que l'on fait ces mêmes régressions pour les variables à expliquer $\bar{h} o(25), \bar{h} o(50)$ et $\bar{h} o(75)$ on constate que :

la première variable introduite demeure l'altitude.

le vent est introduit au deuxième palier pour ho (75) et ho (50) et il est significatif, tandis que pour $\bar{h} o(25)$ il n'intervient qu'au dernier palier et il n'est plus significatif.

lapport du substratum devient significatif pour les trois variables expliquées; introduction au palier 2 pour $\bar{h} o$ (25) et au palier 3 pour $\bar{h} o$ (50) et $\bar{h} o$ (75).

l'exposition intervient significativement au troisième palier pour ho (25) et remplace le vent.

l'explication de la hauteur dominante devient meilleure dans son ensemble :

ho $\left(75: R^{2}=0,824, s=2,567 \mathrm{~m}\right.$,

ho $(50): R^{2}=0,766, s=2,451 \mathrm{~m}$,

ho $(25): R^{2}=0,666, s=1,648 \mathrm{~m}$.

Dans une deuxième série de régressions, nous avons expliqué $H o$ et $\bar{h} o$ après transformation logarithmique comme précédemment par des variables du milieu, à l'exception de la roche-mère, et les variables du peuplement telles que le volume total $\left(V+V^{\prime}+V^{\prime \prime}\right)$, ainsi que $V, N, G, C o, C g, A o, A_{1: 3}, C$ max et $C$ min du peuplement principal et on constate que :

- la première variable introduite est $\left(V+V^{\prime}+V^{\prime \prime}\right)$ suivie de l'altitude,

- viennent ensuite les variables $N, V$, (vent +1$)$ et $G$,

- l'apport de ces 6 variables est significatif à l'exception de la variable $\left(V+V^{\prime \prime}+V^{\prime \prime}\right)$ qui n'est plus significative au $6^{\circ}$ palier,

- pour $\mathrm{Ho}$ le coefficient de corrélation multiple $R^{2}=0,984$ et l'écart-type résiduel $s=0,038$ pour les variables transformées.

Dans une troisième série de régressions, nous avons expliqué $V$ et $\left(V+V^{\prime}+V^{\prime \prime}\right)$ par les variables du milieu et de $A_{0}$ et $A_{130}$ pour chacun des trois substrata et pour l'ensemble. Là aussi, on constate l'influence significative de l'altitude et du vent.

Les résultats de ces différentes régressions nous ont conduit à ne retenir, comme variable d'intérêt forestier définissant le milieu, que l'altitude. L'abandon des autres variables est justifié, soit par leur influence non significative ou leur 
apport très faible, soit par le fait qu'elles ne se présentent pas sous une forme quantitative ou qu'elles sont très mal réparties à l'intérieur de notre échantillon de placettes, comme par exemple, les phonolites que l'on ne trouve qu'au-dessus de $1400 \mathrm{~m}$ d'altitude.

\subsection{3. - Relation hauteur dominante-Altitude}

Nous avons finalement retenu un ajustement polynomial du troisième degré de la forme :

$$
\begin{aligned}
& Y=b_{0}+b_{1} X_{1}+b_{2} X_{1}^{2}+b_{3} X_{1}^{3} \\
& Y=\bar{h} o(25), \bar{h} o(50), \text { et } h o(75) \\
& X_{1}=\text { altitude } \cdot 10^{-3} .
\end{aligned}
$$

Les résultats de cet ajustement sont donnés dans le tableau 10.

\section{TABLEAU 10}

\begin{tabular}{|c|c|c|c|c|c|c|}
\hline \multirow[t]{2}{*}{$Y$} & \multicolumn{2}{|c|}{$\bar{h}_{0}(25)$} & \multicolumn{2}{|c|}{$\bar{h}_{0}(50)$} & \multicolumn{2}{|c|}{$\bar{h}_{0}(75)$} \\
\hline & $\begin{array}{c}\text { Coefficients } \\
b_{0} \text { à } b_{3}\end{array}$ & $t\left(b_{i}\right)$ & $\begin{array}{c}\text { Coefficients } \\
b_{0} \text { à } b_{3}\end{array}$ & $t\left(b_{i}\right)$ & $\begin{array}{c}\text { Coefficients } \\
b_{0} \text { à } b_{3}\end{array}$ & $t\left(b_{i}\right)$ \\
\hline & $-20,4709$ & - & $-43,9506$ & - & $-44,5622$ & - \\
\hline$x$ & 40,5842 & $2,38^{* * *}$ & 84,7004 & $3,21 * *$ & 95,0609 & $3,47^{* *}$ \\
\hline$x^{2}$ & - & - & - & - & - & - \\
\hline$X^{3}$ & $-10,4769$ & $3,37^{* *}$ & $-21,1022$ & $-4,38^{* * *}$ & $-24,0364$ & $4,82^{* * *}$ \\
\hline$R^{2}$ & \multicolumn{2}{|c|}{0,731} & \multicolumn{2}{|c|}{0.797} & \multicolumn{2}{|c|}{0,869} \\
\hline$S \bar{y}$ & \multicolumn{2}{|c|}{$1,438 \mathrm{~m}$} & \multicolumn{2}{|c|}{$2,227 \mathrm{~m}$} & \multicolumn{2}{|c|}{$2,226 \mathrm{~m}$} \\
\hline$\frac{s \bar{y}}{\bar{y}} 100$ & \multicolumn{2}{|c|}{$18,8 \%$} & \multicolumn{2}{|c|}{$13,4 \%$} & \multicolumn{2}{|c|}{$9,9 \%$} \\
\hline
\end{tabular}

Ajustement de la hauteur dominante $\left(\bar{h}_{0}\right)$ en fonction de l'altitude, coefficients $R^{2}$ et écart-type résiduel

* significatif au seuil de $5 \%$

** significatif au seuil de $1 \%$

*** significatif au seuil de $0,1 \%$

L'introduction du vent comme variable supplémentaire n'est pas significative, quoique on diminue légèrement l'écart-type résiduel de la variable ho (75). Il faut cependant faire les quelques remarques suivantes sur ces résultats :

- la forme de la courbe traduit de façon satisfaisante les observations faites sur le terrain, c'est-à-dire, qu'il existe un très faible optimum vers $1150 \mathrm{~m}$ d'altitude ; la hauteur dominante diminue légèrement aux altitudes plus basses. 
- les trois hauteurs dominantes deviennent nulles sensiblement à une même altitude entre 1637 et $1691 \mathrm{~m}$, ce qui correspond à la limite supérieure de la forêt dans la région du Mont Mézenc (1 $754 \mathrm{~m}$ ).

- l'influence de l'altitude augmente à âge croissant et on peut supposer qu'elle exerce un effet cumulatif.

- $87 \%$ de la variation de la hauteur dominante à 75 ans s'explique par l'altitude.

- la hauteur dominante ne varie que très peu entre 1000 et $1400 \mathrm{~m}$ d'altitude pour diminuer rapidement au-delà. Ceci permet de fixer une limite supérieure pour un reboisement rentable.

Dans la figure 4 , nous avons tracé ces trois courbes et placé les valeurs mesurées avec un signe différent pour chacune des trois roches-mères. La répartition des points autour de la courbe laisse supposer une hauteur dominante légèrement plus élevée pour les placettes sur roche-mère granitique que sur roche-mère basaltique, mais la taille de l'échantillon ne permettait pas une discrimination rigoureuse. Une étude des sols et de la nutrition (analyse foliaire) est en cours en collaboraation avec M. F. LE TACON, de la Station des Sols Forestiers du CNRF.

\subsection{4. - Croissance juvénile et altitude}

L'équation 1 nous permet de calculer pour chaque arbre dominant l'âge à $1,30 \mathrm{~m}$ que nous appelons $A 130$. La différence $\triangle A=A_{\mathrm{a}}-A 130$ (âge mesuré à la souche $-A 130$ ) nous indique done le nombre d'années nécessaires pour atteindre la hauteur de $1,30 \mathrm{~m}$.

Nous avons cherché s'il existait une liaison de $\Delta A$ avec l'altitude ct si le substratum avait une influence significative.

Nous avons obtenu l'ajustement suivant :

$$
\begin{aligned}
& y=1099,6348-1,7114 \times \times 0,0007 \mathrm{x}^{2} \\
& R^{2}=0,713 \quad S=17,15 \\
& y=\Delta A .10 \\
& x=\text { altitude en mètres. }
\end{aligned}
$$

La régression polynomiale du degré 3 n'a pas apporté d'amélioration. Ce résultat (fig. $6 a$ ) concorde parfaitement avec les observations et mesures que nous avons faites dans les jeunes plantations d'Épicéa, et confirme une influence croissante de l'altitude avec lâge, comme nous l'avons déjà constaté dans le chapitre 3.13 . La représentation graphique ne laisse pas supposer une influence notable du substratum.

\subsection{5. - Relations circonférence-âge et circonférence-altitude}

A partir de 4 rayons mesurés d'année en année à l'aide de la machine Eklund (PARDÉ, 1958), sur des rondelles découpées à $1,30 \mathrm{~m}$, sur les deux arbres 


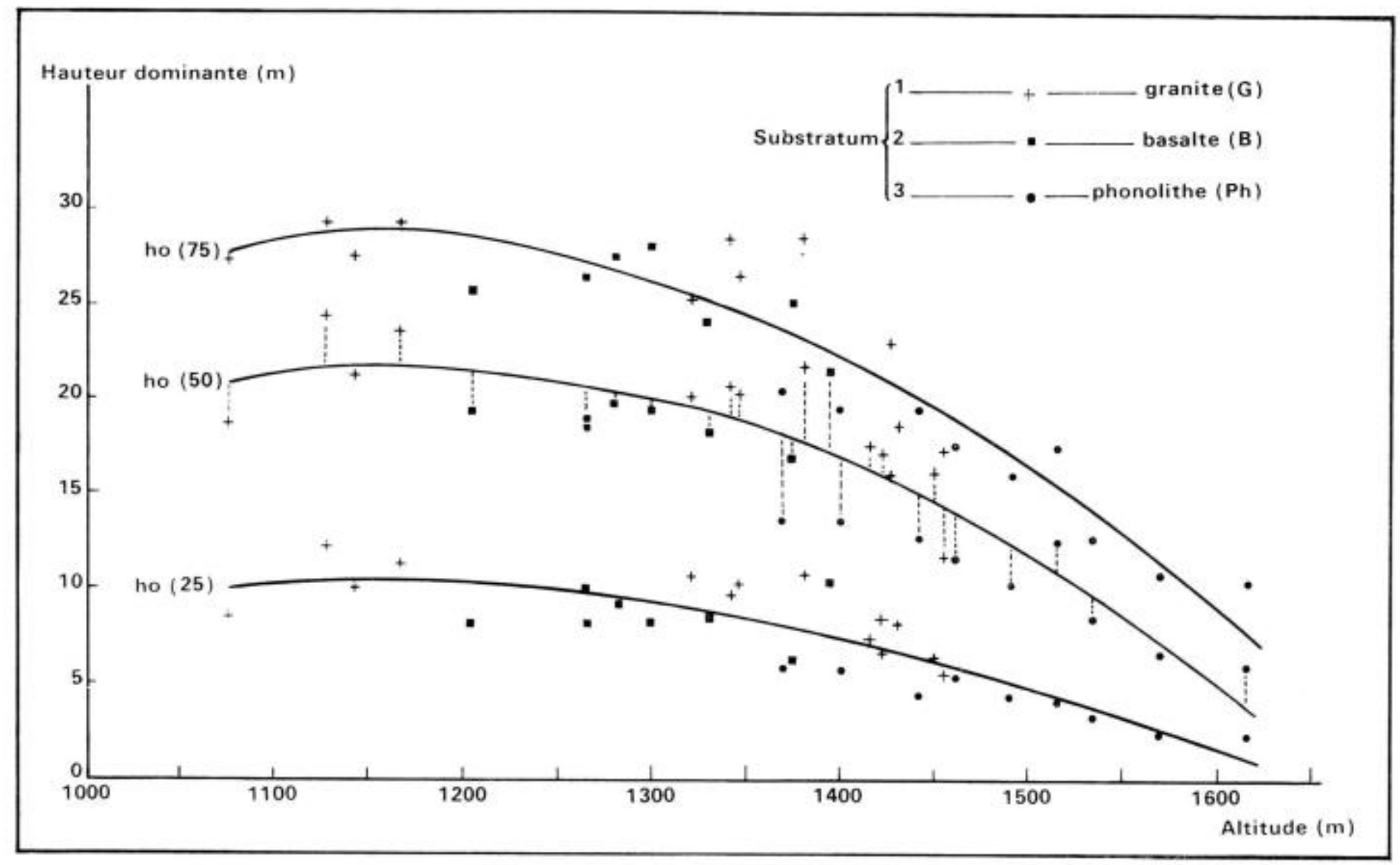

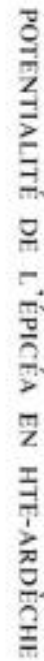

FIG. 4. - Ajustement de la hauteur dominante (ho) en fonction de Paltitude avec représentation des roches-mères 
dominants, nous avons d'abord calculé un rayon moyen (moyennes quadratiques) par arbre d'après la méthode préconisée par Siostrzonek (1958). L'ajustement du rayon moyen en fonction de l'âge à l'aide d'un polynôme du $3^{\circ}$ degré a donné de très bons résultats $\left(R^{2}: 0,99, s=2 \mathrm{~mm}\right)$. Nous avons cependant remarqué une variation cyclique des écarts autour de la courbe d'ajustement, concernant toujours les mêmes années. Nous envisageons une analyse plus détaillée de ce phénomène cyclique à une date ultérieure. Nous avons calculé ensuite les circonférences moyennes sous écorce de deux arbres dominants, pour les âges $25-50$ et 75 ans, que nous appelons $\overline{\mathrm{Co}}(25), \overline{\mathrm{Co}}(50)$ et $\overline{\mathrm{Co}}(75)$.

Il existe évidemment une bonne liaison statistique entre la circonférence dominante (Co) et la hauteur dominante $(H \mathrm{o})$ l'introduction supplémentaire de la densité du peuplement sous la forme $10^{4} / \mathrm{N}+N^{*}$ améliore sensiblement cette liaison tandis que lâge dụ peuplement $(A 0)$ n'a pas d'effet significatif.

$$
\begin{aligned}
& Y=65,9448+1,8205 X_{1}+1,2526 X_{1} \\
& R^{2}=0,67 s y=10,45 \mathrm{~cm} \bar{Y}=127,15 \mathrm{~cm} \\
& Y=C o \text {-circonférence dominante sur écorce }(\mathrm{cm}) \\
& X_{1}=H \mathrm{o} \text {-hauteur dominante }(\mathrm{m}) \\
& X_{3}=10^{4} / N+N^{\prime} ; N+N^{\prime} \text { (nombre de tiges à l'hectare) }
\end{aligned}
$$

De même, la circonférence dominante $(\mathrm{Co})$ et la circonférence moyenne du peuplement $\mathrm{Cg}$ sont liées linéairement. L'introduction de la densité comme précédemment diminue l'écart-type résiduel $(s \bar{y})$ de plus de $50 \%$. L'âge n'intervient pas significativement.

$$
\begin{aligned}
& \frac{Y}{Y}=8,2060+0,5140 X_{1}+1,5011 X_{2} \\
& y=96,93, R^{2}=0,95 s y=3,7 \mathrm{~cm} \\
& Y=C_{g}: \text { circonférence moyenne }(\mathrm{cm}) \\
& X_{1}=\mathrm{Co}: \text { circonférence dominante }(\mathrm{cm}) \\
& X_{2}=10^{4} / N+N^{\prime} \text { densité du peuplement. }
\end{aligned}
$$

L'ajustement de la relation circonférence - altitude sur un polynôme du $3^{\circ}$ degré, donne des résultats satisfaisants, mais la dispersion autour de la courbe est beaucoup plus grande que dans le cas des hauteurs dominantes (fig. 5).

Les équations étaient de la forme :

$$
Y=C o+C_{1} X+C_{2} X^{2}+C_{3} X^{3}
$$

$Y=$ circonférence moyenne (en mm) des deux arbres dominants sous écorce pour les âges 25,50 et 75 .

$$
X=\text { altitude } \cdot 10^{-3} \text {. }
$$

Les coefficients, les $R^{2}$ et les écarts-types résiduels sont donnés dans le tableau 11 et nous pouvons faire les remarques suivantes :

- à âge égal, les circonférences diminuent moins rapidement avec l'altitude que les hauteurs dominantes; la forme de la courbe est sensiblement la même pour les 3 âges. N. MaIr (1967) a constaté dans les Alpes du Tyrol, que l'accroissement en diamètre reste relativement élevé même à la limite supérieure de la forêt ; il ne diminue qu'à un âge très avancé.

- l'introduction des variables du milieu, comme la pente, l'exposition et le vent n'est pas significative et ne diminue pas l'écart-type résiduel. 


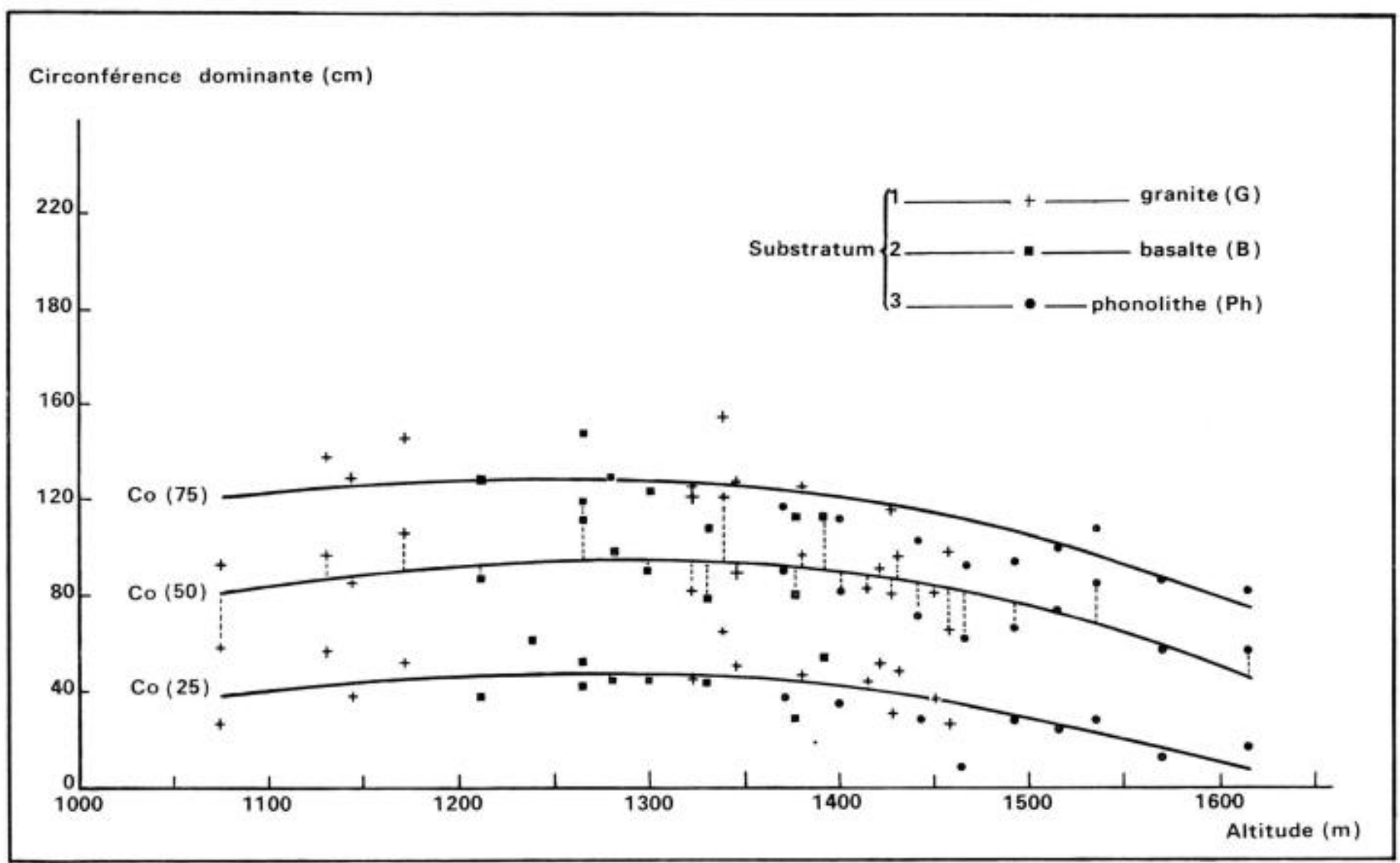

FIG. 5. - Ajustement de la circonférence dominante sous écorce en fonction de l'altitude avec représentation des roches mères 


\section{TABLEAU 11}

Ajustement de la circonference dominante $\left(\bar{c}_{0}\right)$ en fonction de l'altitude, coefficients $R^{2}$ et écart-type résiduel

\begin{tabular}{|c|c|c|c|c|c|c|}
\hline \multirow[t]{2}{*}{$Y$} & \multicolumn{2}{|c|}{$\bar{C}_{0}(25)$} & \multicolumn{2}{|c|}{$\overline{C_{0}}(50)$} & \multicolumn{2}{|c|}{$\bar{C}_{0}(75)$} \\
\hline & $\begin{array}{l}\text { Coefficients } \\
c_{0} \text { à } c_{3}\end{array}$ & $t(n)$ & $\begin{array}{l}\text { Coefficients } \\
\text { coà cs }\end{array}$ & $t(c))$ & $\begin{array}{c}\text { Coefficients } \\
\text { coà ca }\end{array}$ & $f(c i)$ \\
\hline & $-2598,3696$ & - & $-3454,0054$ & - & $-1915,5781$ & $2,29 * 0$ \\
\hline$x$ & 3676,6215 & $3,06 * *$ & 5182.0966 & $3,30^{* *}$ & 3902,1914 & - \\
\hline$X^{2}$ & - & - & - & - & - & - \\
\hline$X: 1$ & $-777,9592$ & $3,5,4 *$ & $-1057,7443$ & $3,69 * 4 *$ & $-861,2536$ & $2,77^{* *}$ \\
\hline$R^{2}$ & 0.493 & - & 0,449 & - & 0,514 & - \\
\hline$s \bar{y}$ & 104,41 & - & 132.58 & - & 138,68 & - \\
\hline$\frac{s \bar{y}}{\bar{y}} 100$ & $26,5 \%$ & - & $15,4 \%$ & - & $11,9 \%$ & - \\
\hline
\end{tabular}

* Significatif au seuil de $5 \%$

** Significatif au seuil de $1 \%$

$* *$ Significatif au seuil de $0,1 \%$.

\subsection{6. - Relation nombre de tiges à l'hectare - hauteur dominante}

Il existe une assez bonne relation entre la densité et la hauteur dominante, mais elle n’est pas indépendante de l'âge. Si pour la majorité de nos placettes la densité peut être considérée comme très proche de la densité maximum de la station, il y a cependant certaines placettes dans lesquelles les chablis de l'hiver 1957-58 avaient sérieusement diminué le nombre de tiges à l'hectare.

Nous avons retenu l'équation suivante pour les 33 placettes :

$$
\begin{aligned}
& L N\left(N+N^{\prime}\right)=13,0285-0,8413 L N\left(H_{0}\right) \ldots \ldots \ldots \ldots(3) . \\
& R=0,51 \quad s=0,364 .
\end{aligned}
$$

La densité moyenne de plantations récentes varie entre 3600 et 2400 tiges

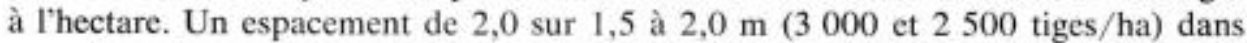
une plantation en ligne, semble donner satisfaction à tout point de vue. La densité initiale de nos placettes était certainement beaucoup plus élevée (de l'ordre de 10000 plants à l'hectare). La figure $6 b$ représente le nombre de tiges à l'hectare en fonction de l'altitude d'après les formules (2) et (3).

\subsection{7. - Relation hauteur dominante - production}

Cette relation qui est valable pour une essence et une région climatique données a été souvent vérifiée (MOOSMAYER, 1957). 
En fait, nous ne connaissons pas la production totale depuis l'origine, faute de dispositifs permanents, et de l'évaluation exacte des volumes enlevés en éclaircie, mais nous savons que pour l'ensemble de nos placettes, les éclaircies antérieures à 1957 étaient très faibles ou nulles et elles correspondent grossièrement à la mortalité naturelle.

Notre relation $\left(V+V^{\prime}+V^{\prime \prime}\right)=f\left(H_{0}\right)$ sous-estime donc la production totale, ceci n'a que peu d'influence pour les peuplements d'altitude mais présente certains inconvénients pour des peuplements auxquels on veut appliquer une sylviculture intensive.

Nous avons obtenu, après transformation logarithmique (logarithme Népérien) l'équation suivante :

$$
\begin{aligned}
& L N\left(V+V^{\prime}+V^{\prime \prime}\right)=-3,0931+1,2491 \quad L N(H o) \\
& R=0,923 \quad s=0,134 .
\end{aligned}
$$

Ce qui correspond à une erreur-type de $103,3 \mathrm{~m}^{3}$ en valeur absolue et de $13,6 \%$ en valeur relative pour les données non transformées. Les deux moyennes ne sont pas significativement différentes.

L'explication de la production totale en fonction de la hauteur dominante peut être légèrement améliorée par l'introduction supplémentaire de l'âge $\left(A_{0}\right)$ et de la densité $\left(N+N^{\prime}\right)$; les trois variables restent significatives, et nous obtenons l'équation suivante :

$$
\begin{aligned}
& L N\left(V+V^{\prime}+V^{\prime \prime}\right)=-5,9253+1,3226 \quad L N \quad(H o)+0,1461 L N\left(N+N^{\prime}\right) \\
& +0,3037 L N(A o) \ldots \ldots \ldots \ldots \ldots \ldots \text { (4) } \\
& R^{2}=0,889 \quad s=0,119 .
\end{aligned}
$$

Nous avons employé les relations 2,3 et 4 pour calculer une estimation de la production totale à l'âge de 75 ans en fonction de l'altitude. Les résultats sont donnés dans la figure $6 c$ et le tableau 12 .

\begin{tabular}{|c|c|c|c|}
\hline $\begin{array}{l}\text { Altitude } \\
\text { (m) }\end{array}$ & $\begin{array}{c}\text { Hauteur } \\
\text { dominante } \\
(\mathrm{m})\end{array}$ & $\begin{array}{c}\text { Production } \\
\text { totale } \\
\mathrm{m}^{3} / \mathrm{ha} \text { ) }\end{array}$ & $\begin{array}{c}\text { Accr. moy. } \\
\left(\mathrm{m}^{3} / \mathrm{ha}^{-1} \text { années }{ }^{-1}\right)\end{array}$ \\
\hline 1000 & 26,46 & 849 & 11.3 \\
\hline 1050 & 27,43 & 886 & 118 \\
\hline 1100 & 28,01 & 909 & 12,1 \\
\hline 1150 & 28,20 & 916 & 12.2 \\
\hline 1200 & 27,98 & 908 & 12,1 \\
\hline 1250 & 27,32 & 882 & 11,8 \\
\hline 1300 & 26,21 & 839 & 11,2 \\
\hline 1350 & 24,63 & 779 & 10,4 \\
\hline 1400 & 22,57 & 701 & 9,3 \\
\hline 1450 & 20,00 & 607 & 8.1 \\
\hline 1500 & 16,91 & 496 & 6,6 \\
\hline 1550 & 13,28 & 371 & 4,9 \\
\hline 1600 & 9,08 & 235 & 3,1 \\
\hline 1650 & 4,31 & 96 & 1,3 \\
\hline
\end{tabular}

\section{Tableau 12}

Hauteur dominante, production totale et accroissement moyen à rage de 75 ans en fonction de l'altitude. 


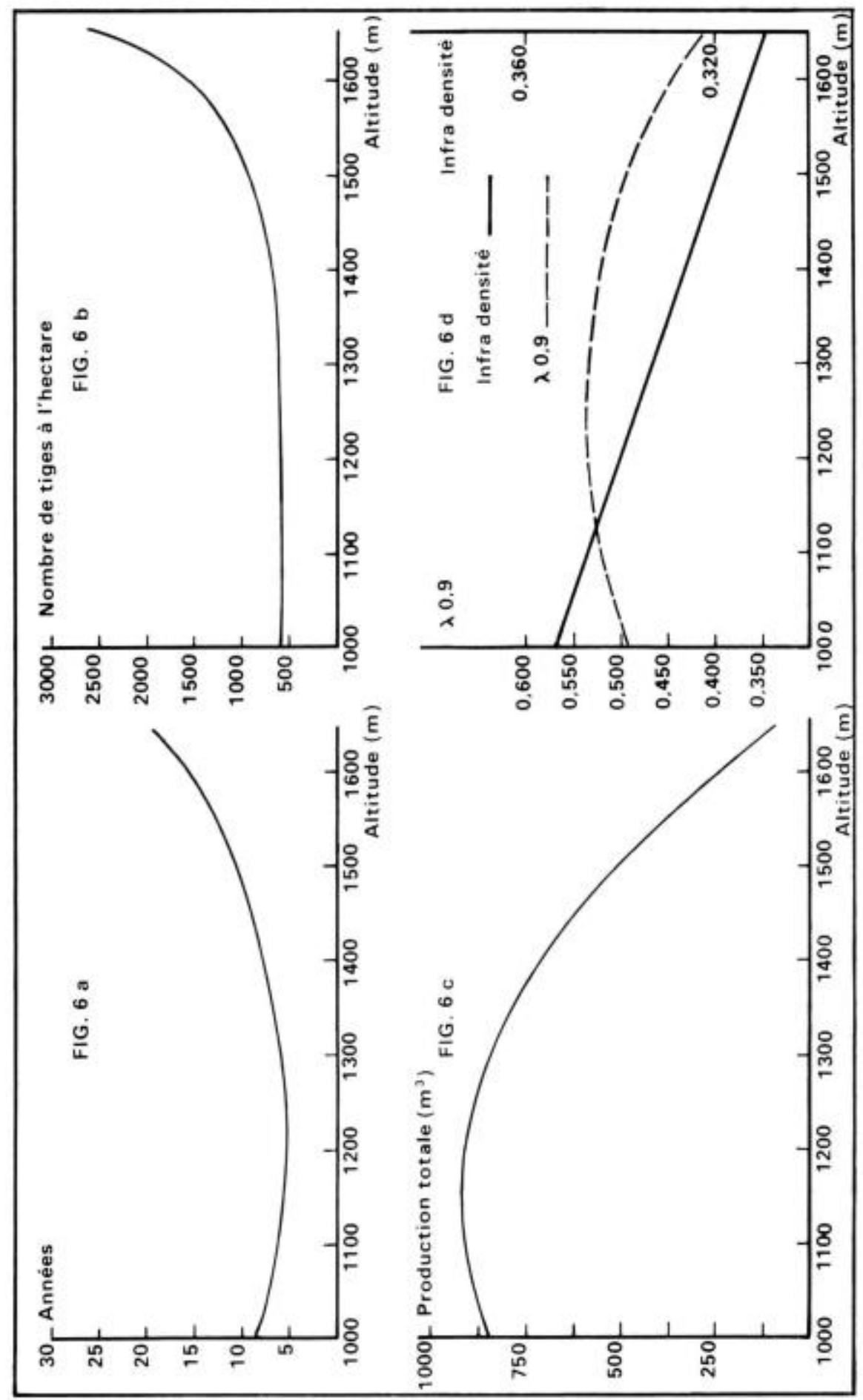

FiG. 6a. - Croissance juvénile et altitude. L'ordonnée représente le nombre d'années pour atteindre la hauteur de $1,30 \mathrm{~m}$

Fig. $6 b$. - Nombre de tiges à lhectare $\left(N+N^{\prime}\right)$ en fonction de l'altitude, pour lâge de 75 ans

FIG. $6 c .-$ Estimation de la production totale $\left(V+V^{\prime}+V^{\prime \prime}\right)$ en fonction de Taltitude pour lâge de 75 ans

Fig. $6 d$. - 20,9 en fonction de laltitude pour un âge de 75 ans et une pente moyenne de $10^{\circ}$, ainsi que linfra-densité $d u$ bois en fonction de l'altitude pour un âge de 75 ans 
La hauteur dominante $(H o)$ et la hauteur moyenne du peuplement $(H \mathrm{~g})$ sont très étroitement liées. Cette relation n'est que très faiblement améliorée pour l'introduction supplémentaire de la densité et de lâge dont seule la densité est faiblement significative.

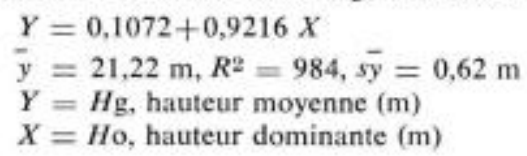

\subsection{8. - Les coefficients de forme et laltitude}

Dans un premier stade, nous avons calculé le coefficient de forme $F_{\text {no }}$ du peuplement (Prodan M., 1965).

$$
F_{130}=\frac{V}{G \cdot H_{g}}
$$

La dispersion est telle qu'aucune liaison, ni avec l'altitude, ni avec des paramètres du peuplement (âge, hauteur, circonférence), n'a pu être mise en évidence. Pour les 33 peuplements, nous obtenons un coefficient de forme moyen de 0,4970 avec un écart-type de la distribution de $0,027(5.4 \%)$. En connaissant $\mathrm{N}, \mathrm{C}_{\mathrm{s}}, \mathrm{H}_{\star}$ et $\mathrm{F}_{130}$, on obtient une estimation du volume sur pied (PARDÉ J., 1963).

Nous avons calculé le volume estimé du peuplement principal avec la valeur moyenne de $F_{1 x}=0,4970$. La différence entre le volume mesuré $(V)$ et le volume estimé $(V)$ n'est pas significative. L'erreur-type en valeur absolue est de $29,35 \mathrm{~m}^{3}(5,5 \%$ en valeur relative).

Dans un deuxième stade, nous avons calculé pour chacun des deux arbres dominants, un facteur de réduction $\lambda_{0,9}$ qui traduit la forme géométrique d'un arbre et permet des comparaisons de forme (J. PARDÉ, 1961). Nous avons expliqué la valeur moyenne des deux arbres en fonction des variables du milieu. Nous avons retenu la régression suivante :

$$
\begin{aligned}
& Y=8,2015+68,8150 x_{1}^{2}-36,0246 x_{1}^{3}+0,1725 x_{2}+0,0963 x_{3} \\
& R^{2}=0,602 \quad s \bar{y}=2,326(5,2 \%) \\
& Y=\lambda_{0,9} \cdot 10^{2} \\
& x_{1}=\text { altitude } \cdot 10^{-3} \text { en mètres } \\
& x_{2}=\text { pente en degrés } \\
& x_{3}=\text { âge. }
\end{aligned}
$$

Les 3 variables explicatives sont significatives.

Dans la figure $6 d$, nous avons présenté l'estimation de $\lambda_{0,9}$ pour un âge de 75 ans et une pente moyenne de $10^{\circ}$. Sans vouloir trop insister sur les valeurs absolues des $\lambda_{0,3}$, nous constatons également une diminution avec l'altitude. En fait, la dispersion est telle que jusqu'à une altitude de $1500 \mathrm{~m}$, le $\lambda_{0,0}$ ne varie pratiquement pas avec l'altitude et, ce n'est qu'au-delà de ce seuil qu'on observe une brusque diminution de ce coefficient. Une estimation de $\lambda_{0}$ à 
partir de $\mathrm{Ho}$ et $\mathrm{Co}$, donne un ajustement légèrement meilleur mais ne change pas l'allure de la courbe.

\subsection{9. - L'inira-densité du bois et laltitude}

La diminution de la densité du bois à altitude et latitude croissante, est généralement un fait reconnu. En outre, il existe une liaison négative, étroite entre la densité et la largeur des cernes, ainsi qu'une liaison positive avec l'âge. Toute chose égale par ailleurs, la densité dépend également du pourcentage du bois d'été (A. BERNHART, 1964).

Nous avons prélevé une carotte à $1,30 \mathrm{~m}$ dans les 4 plus gros arbres de chaque placette, dans une direction choisie au hasard.

Pour les 30 premières années, à partir de 1965, la Station des Recherches sur la Qualité des Bois a effectué la détermination de l'infra-densité, qui est le rapport du poids anhydre sur le volume saturé (Polge, 1963).

Nous avons expliqué la densité moyenne de ces 4 échantillons en fonction de l'altitude et de l'âge du peuplement.

$$
\begin{aligned}
& Y=324,0542-69,8990 X_{1}+1,3325 X_{2} \\
& R_{2}=0,53 \quad s_{y}^{-}=18,368 \\
& Y=\text { densité moyenne } \cdot 10^{-7} \\
& X_{1}=\text { altitude }(\mathrm{m}) \cdot 10^{-3} \\
& X_{2}=\text { âge (années) du peuplement. }
\end{aligned}
$$

Sans vouloir insister sur les valeurs absolues de cette liaison, nous pouvons confirmer, qu'à toutes autres choses égales, la densité diminue linéairement avec l'altitude.

Dans la figure $6 d$, nous avons représenté la densité en fonction de l'altitude pour un âge de 75 ans.

\section{2. - OBSERVATIONS SUR LES ASSORTIMENTS DE PRODUITS ET SUR l.A VALEUR SUR PIED DES PEUPLEMENTS}

Dans notre étude, nous avons toujours exprimé la production en volume bois-fort sur écorce, or un même volume sur pied peut être composé de tiges de taille et de qualité fort différentes, et en conséquence, avoir une valeur sur pied très variable.

La classification des grumes (VENET J., 1951) peut s'appliquer aux peuplements sur pied à partir d'une étude détaillée (DECourT N., 1967), Le calcul automatique du volume des assortiments et de la valeur sur pied est réalisable (SchöPfER W., 1966). A la Station de Sylviculture et de Production, un programme de recherche est actuellement en cours de réalisation dans ce but.

Dans notre étude, nous étions limités aux observations suivantes :

Dans les peup'ements, âgés d'environ 75 ans, des grumes de * qualité exceptionnelle s et de * choix I et II s sont très rares et la quasi totalité est 
constituée par le * choix III et IV \$, mais dans des peuplements denses et jusqu'à une altitude d'environ $1350 \mathrm{~m}$ on trouve une quantité élevée de poteaux de bonne qualité.

Entre $1350 \mathrm{~m}$ et $1450 \mathrm{~m}$ d'altitude, la qualité des assortiments diminue très rapidement suivant l'exposition au vent, et au-delà de $1450 \mathrm{~m}$ on ne peut guère compter que sur une \&qualité chauffages quoique la production puisse être encore non négligeable. Le choix d'une limite altitudinale de reboisement doit donc tenir compte non seulement de la production en volume bois-fort tige ou autre, mais également de la répartition des produits et de leurs valeurs.

\section{3. - OBSERVATIONS SUR LA SYLVICULTURE ACTUELLE ET FUTURE}

La plupart des peuplements d’épicéa sont aménagés en futaie jardinée avec des possibilités fixées soit par volume, soit par contenance. Pourtant, les interventions sylvicoles n'ont pas encore permis d'établir une structure véritablement jardinée : tous les peuplements ont conservé leur aspect régulier.

Mais il existe encore de nombreuses séries domaniales de reboisement non aménagées et il faut se demander si le jardinage pied par pied ou même par bouquet - en ce qui concerne les peuplements purs d'Épicéa dont la majorité dépasse un âge de 80 ans — n'entraverait pas la stabilité des peuplements sans pour autant pouvoir assurer l'avenir.

En effet, la régénération naturelle ne semble possible, avec un succès d'ailleurs variable, que sur certaines stations entre 1000 et $1350 \mathrm{~m}$ d'altitude, elle est surtout liée à la présence du Sapin pectiné, tandis qu'aux stations plus élevées et dans des peuplements purs d'Épicéa, la régénération naturelle est gravement compromise même si on acceptait une période de mise en régénération abusivement longue.

La stabilité des peuplements est précaire. Les interventions sylvicoles notamment les éclaircies, sont trop rares et surtout trop tardives; les provenances d'Ép.céa utilisées, sont généralement inconnues et parfois mal adaptées. Dans ces conditions, on n'a pu obtenir une structure stable des peuplements permettant de résister au rude climat d'altitude : dans certaines forêts, la moitié de la possibilité annuelle est récoltée sous forme de chablis.

La question des éclaircies ne peut être abordée dans cette étude, faute de connaissances précises sur l'évolution des peuplements. La densité moyenne des peuplements (nombre de tiges et surface terrière à l'hectare) nous semble toutefois un peu au-dessus de l'optimum. Mais le problème ne sera pas le même pour les reboisements récents et futurs plantés à espacements plus grands en utilisant des provenances convenables.

L'état sanitaire des peuplements est dans son ensemble satisfaisant, mais nous avons pu observer dans des peuplements âgés une proportion assez élevée d'arbres atteints par la pourriture rouge du tronc, ce qui obligera à ramener la révolution à 70 ou 80 ans seulement. Peut-être, une deuxième génération sera-t-elle plus résistante vis-à-vis de la pourriture. 
Les résultats des chapitres 3.1 , à 3.3 , et les observations précédentes faites sur le terrain, nous laissent penser qu'à l'avenir le reboisement artificiel et un aménagement en futaie régulière à révolution relativement courte s'imposeront sur les stations à forte productivité.

\section{3. - COMPaRAISONS DES DIVERSES ESSENCES}

Outre les 33 placettes d'Êpicéa, nous avons mesuré une placetie de Sapin pectiné, une placette de Douglas et trois peuplements de Pin sylvestre. Seule la comparaison du Sapin avec l'Epicéa peut se faire sur une base commune et confirme une croissance juvénile plus faible du Sapin et une production totale sensiblement égale à un âge avancé.

En ce qui concerne le Douglas, nous pensons comme d'autres spécialistes du reste, qu'au-dessus de 1000 à $1100 \mathrm{~m}$ d'altitude, il n'est plus à sa place (risques de dégâts dus aux gelées, givre et vent) quoique sa production puisse encore égaler celle de l'Épicéa et du Sapin.

Le Pin sylvestre qui occupe actuellement une place importante dans la forêt privée et l'économie rurale, ne peut pas rivaliser avec l'Épicéa et le Sapin pectiné, ni par sa production (tableau 9), ni par sa qualité et encore moins par ses prix. On peut envisager une substitution d'essence (Sapin-Épicéa) à long terme.

Dans notre étude, nous n'avons pas pu tenir compte du facteur \& provenance * faute de renseignements sur l'origine des graines. L'amélioration des arbres forestiers a fait de grands progrès et en particulier la sélection de l’Épicéa pour les reboisements d'altitude (Bouvarel, 1960); elle a mis à la disposition du reboiseur un certain nombre de provenances d'Épicéa, qui, tant sur le plan production et qualité que sur le plan résistance vis-à-vis des facteurs biotiques et abiotiques, notamment par la sélection d'arbres à cime étroite, permettront un gain substantiel (O.N.F., 1967, LACAZE, 1968).

\section{5. - La validité des résultats a plus grande Échelle : COMPARAISON AVEC 8 PLACETTES D'ÉPICÉA DE LA HAUTE-LOIRE ET DE LA LOIRE MESURÉES EN 1961}

Pour exprimer la croissance en hauteur des arbres dominants d'une manière plus générale, nous avons calculé les coefficients $a_{0}$ à $a_{3}$ de l'équation (1) en fonction de l'altitude, de l'âge et certains termes d'inter-action. Les résultats obtenus ne sont que partiellement satisfaisants; ils ne sont valables que pour un âge de 75 ans environ et des altitudes comprises entre 1000 et $1350 \mathrm{~m}$. En tenant compte de ces restrictions, nous avons calculé les hauteurs dominantes des 8 placettes installées par nos prédécesseurs en dehors et au nord de notre zone d'action actuelle pour un âge de 75 ans. Les différences entre les hauteurs dominantes constatées et celles correspondant aux valeurs calculées (équation 2 tableau 10 et fig. 4) ne sont pas significativement différentes $(P=0,05)$. On peut donc supposer que les différents résultats restent valables dans une région plus vaste couvrant les départements de la Haute-Loire et de la Loire. 


\section{6. - Choix d'une limite altitudinale de Reboisement}

Dans l'état actuel de nos connaissances, nous ne pouvons proposer qu'un choix assez arbitraire d'une zone limite de reboisement en altitude, car outre la production brute, la valeur sur pied et les quelques considérations écologiques, d'autres facteurs tels que le but de production recherché (bois d'industrie ou sciage), l'aménagement et l'organisation économique de la région, ainsi que l'évolution technique ont une influence non négligeable.

Cette zone-limite supérieure, nous la plaçons actuellement dans cette région entre 1400 et $1450 \mathrm{~m}$ d'altitude à l'exclusion toutefois des stations sommitales exposées au vent; elle est valable aussi bien pour l'Épicéa que pour le Sapin pectiné.

\section{CONCLUSION}

Les résultats des précédents chapitres ne sont qu'une première approche du problème des potentialités forestières en Haute-Ardèche et ne concernent que l'Épicéa: ils permettent néanmoins une estimation de la production de cette essence dans une région assez importante et avec une précision suffisante pour une planification régionale. C'est dans un but de simplification que nous avons retenu seulement l'altitude comme variable synthétique pour le climat et le type de sol. Notre échantillon de 33 placettes n'était cependant pas représentatif pour toutes les stations, car nous n'avons pas trouvé de peuplements sur sol hydromorphe, ni sur sol superficiel ou peu profond, mais ces sols sont très peu répandus dans cette région.

Le choix d'une zone entre 1350 et $1450 \mathrm{~m}$ comme limite altitudinale de reboisement peut servir de première indication pour les reboisements futurs, mais il faudra attendre une table régionale de production pour avoir un modèle de sylviculture et une estimation des assortiments des produits : la Station de Sylviculture et de Production s'y emploie dès maintenant.

Dans un stade ultérieur, après un prolongement économique des tables de production, on peut envisager une délimitation objective des zones à vocation forestière, agricole ou autres, à condition de connaître également les potentialités agricoles sous une forme quantitative en fonction des facteurs du milieu et des facteurs techniques.

L'aménagement des peuplements d'Épicéa en futaie régulière, une évolution relativement courte (70 à 80 ans) et la régénération par plantations avec une certaine proportion de sapins en utilisant des provenances adéquates, semblent les mieux adaptés aux conditions de cette région.

Mais le résultat essentiel est certainement la confirmation indiscutable de la forte productivité de l'Épicéa ainsi que du Sapin. L'accroissement moyen de $11 \mathrm{~m}^{3}$ par hectare et par an à l'âge de 75 ans sur les hauts plateaux de l'Ardèche et de la Haute-Loire montre que cette région est à vocation essentiellement forestière. 


\section{REMERCIEMENTS}

Que soient remerciés ici :

- les propriétaires qui nous ont autorisé à installer chez eux nos placettes,

- l'Office National des Forêts (Centres de Gestion d'Aubenas et du Puy) en tant que propriétaire, ainsi que pour laide que nous ont apporté ses agents,

- les membres de la Chambre d'Agriculture de l'Ardèche notamment M. Pourrat,

- M. Parde, Directeur de Recherches, Chef de la Station de Sylviculture et de Production, pour ses bons et nombreux conseils, boration,

- MM. Aussenac, Decolrt, Le TACon et Tomassone pour leur étroite collaet secrétaires.

\section{SUMMARY}

\section{POTHNTIAL PRODUCTIVITY OF NORWAY-SPRUCE (Picéa A bies L.) IN THE FRENCH} CENTRAL MASSIF (ARDÈCHE)

The article concerns the variation of the production of Norway-spruce (Picea Abies L.) as a function of environment in the elevated plaines (above $1000 \mathrm{~m}$ above sea level) of the French Central Massif (Ardèche and Haute-Loire).

More than 40 stands and plantations (non-permanant sample-plots with stem-analysis) were studied by multiple regression methods. Among the different variables which were analyzed in detail (altitude, soil, exposition, slope etc.) altitude alone was responsible for $87 \%$ of the total variation of the dominant stand height at age 75.

The results obtained are confirming the high productivity of Norway-spruce in that region and permits the choice of an upper limite of reafforestation (1400 to 1450 meters) with regard to actual economic conditions. Some observations concerningg regional Norwayspruce - silviculture are furnished.

\section{ZUSAMMENFASSUNG}

\section{die potentielle WuChsleistung der fichte (Picea Abies L.) IM Zentralmassiv} (HAUTE ARDËCHE)

Die vorliegende Arbeit betrifft die Variation der Wuchsleistung der Fichte (Picea Abies L.) als eine Funktion des Standortes auf den in über $1000 \mathrm{~m}$ Seehöhe gelegenen Hochplateaux der Departements Ardèche und Haute Loire im französischen Zentralmassiv.

Mehr als 40 gleichaltrige Reinbestände und Aufforstungen (einmalig aufgenommene Versuchsflächen mit Stammanalysen) wurden statistisch untersucht (Mehrfachregressionsanalysen). Unter den im Detail analysierten Faktoren, wie Sechöhe, Grundgestein, Exposition, Hangneigung etc. werden allein mit der Seehöhe 87 \% der Variation der Bestandesoberhöhe im Alter 75 erklärt.

Die vorliegenden Ergebnisse bestätigen die hohe Wuchsleistung der Fichte im Untersuchungsgebiet und erlauben eine, den aktuellen Wirtschaftsbedingungen entsprechende, obere Aufforstungsgrenze $(1400-1450 \mathrm{~m})$ festzulegen. Desweiteren wurden Beobachtungen über den regionalen Fichtenwaldbau gemacht.

\section{REFERENCES BIBLIOGRAPHIQUES}

AssmanN E., 1967. Konsequenzen neuer ertragskundlicher Erkenntnisse für die Forsteinrichtung. Wiss. Z. Techn, Univers. Dresden, 16, 5, 1559-1565.

BALSEINTE R.. 1966. Climats montagnards et stations climatiques d'altitude en France, 523 p. Thèse, Doct. Lettres. Fac, Lettres Sci, humaines Grenoble. 
Beille L., 1889. Essai sur les zones de végétation du Massif Central de la France. 153 p. Bull, Soc. Sci, phys, nat. Toulouse,

BERnharT A., 1964. Waldbauliche Möglichkeiten einer Beeinflussung der Rohdichte von Fichtenholz. Mitt. Staatsforstverwaltung Bayerns, 34, 132-141.

Bouvarel. P., 1960. Die französischen Fichtenherkünfte für die Gebirgsaufforstung. Dans Forstssamengewinnung und Pflanzenanzucht für das Hochgebirge, 41-53 Bayrischer Landwirtschaftsverlag Münich.

Buffault P., 1916, Les reboisements du Mézenc, Rev. Eaux et Forêtts, 153-161.

Carles J., 1953. A travers le Velay. Bull. Soc, bot. Fr., 100, 59-67.

Carles J., 1956. Carte de la végétation de la Francé au 200000 ; Notice détaillée de la feuille 59, Le Puy. C.N.R.S., 40 p.

Coutagne A., 1954, Hydrométéorologie de bassins de haute montagne, S.H.F., Paris, 2 vol.

Curtis R.O., 1964. A stem-analysis approach to site-index curves. Forest. Sci., 10, 2, 241-256.

Daget Ph., Poissonet J., 1966. Etude phyto-écologique de la commune de Thoras (Haute-Loire), C.N.R.S. - C.E.P.E. - Doc. $\mathrm{n}^{*} 28,5$ fasc. ronéo.

Decourt N., 1966. Instruction pour l'assiette et les mensurations des placettes temporaires et semi-permanentes. Station de Sylviculture et de Production. C.N.R.F., note interne,

Decourt N.. 1967, Le Douglas dans le Nord-Est du Massif Central - Tables de production provisoires, Ann. Sci. forest., 24, 1, 45-85.

DefFontaines J.P. et Bovgler J., 1965. Etude des polentialités agricoles en HauteArdèche. 166 p. Chambre de l'Agriculture de l'Ardèche et Laboratoire d'Agriculture et de Zootechnie de l'I.N.R.A., ronéo.

Deffontaines J.P., 1967. Une méthode de détermination des facteurs techniques limitant la production agricole en montagne, Fourrages, 31, 36-52.

Duchaufour Ph. et Souchier B., 1966. Note sur une méthode d'extraction combinée de raluminium et du fer libres dans les sols. Sci. Sol, 1, 17-29.

Etienne P., 1956. Recherches sur le climat du Massif Central Français, Mémor. Météorol. Nation, (43), p. 242.

Flahault Ch., 1901. Les limites supérieures de la végétation forestières et les prairies pseudoalpines en France. Rev. Eaux et Forêts, 385-439.

Gaillard L.. 1964. Etude historique sur la forêt de Mazan (Ardèche). Rev, forest, franç., $11,831-845$.

GADANT J., 1968b. Le reboisement en Auvergne. Rev, forest. frans., 7/8, 449-457.

Gallots L., 1933. Les pins d'émonde du Forez, du Velay et du Vivarais, Bull. Soc. forest. Franche-Comté, 20, 1, 29-32.

Giмсовне A., 1967. Le coefficient bioclimatique de productivité potentielle. Oréol. Plant., 2, 3, $183-216$.

Guinier Ph., 1956. Arbres et forêts du Massif Central (Notes botaniques et forestières). Bull, Soc. bot. Fr., 203, 95-114.

Jacques P., 1941. Les pins sylvestres dans le Massif Central, Rev, forest, fraņ̧, 100, 711 720.

J.O., 1955. A propos du reboisement: Lettres du Comte de Montlosier au Préfet du Puy-de-Dôme (18-2-1827). J. Off. Républ. franş.

LACAZE J.F., 1968. Un épicéa tardif, Rev, forest. fran ., 6, 401-406.

LEMEE G.+ 1953. Observations sur la végétation actuelle et son évolution postglaciaire dans le Massif du Mézenc. Bull. Soc. bot. Fr., 103, 83-95.

Lemee G. et Carbiener R., 1956, La végétation et les sols des voleans de la chaine des Puys. Bull. Soc, bot. Fr., 103, 7-30.

Le Tacon F. et Oswald H., 1969. Les sols de Haute-Ardèche. Bull. Assoc. Franç. Etude sol (sous presse).

"Gadant J., 1968a). La forêt et le bois en Auvergne. Conseil régional de la forêt et des produits forestiers. Service Régional d'Aménagement forestier, Clermont-Ferrand, 104 p.

Magin R., 1967. Ertragsfestsetzung und Ertragsprognose in der Forsteinrichtung. Mitt. FBVA, Vienne, 77, 1, 209-222. 
Marr N., 1967. Zuwachs und Ertragsleistung subalpineri. Wälder Mitt. FBVA, 75, 383-426.

MiNOR Ch.O., 1964. Site-index curves for young-growth Ponderosa pine in northern Arizona. 8 p. U.S. For. Serv, Res. Note Rocky Mt. For. Range Exp. Sta, n ${ }^{\circ}$ RM 37.

Moosmayer H.U., 1957. Zur ertragskundlichen Auswertung der Standortgliederung im Ostteil der schwäbischen Alp. Mitt. Vereins Fortl. Standortsk. Forstpflanzenzücht. 7, 1-41.

Moret L., 1958. Précis de Géologie. Masson, Paris, 675 p.

NIER, 1952. Historique et avenir des reboisements dans le département de la Loire. Rev. forest, franç., 11, 714-728.

O.N.F., 1967. Le choix des provenances d'épicéas, Circ, Off. nation. Forêts, 67-D22, 19.7.1967.

O.N.M., 1942 a. Etude climatologique du Haut-Languedoc et des Cévennes, 28 p. Off. nation. Météorol. (climatologie) (janvier).

O.N.M., 1942 b. Etude climatologique du Bas-Languedec et du Roussillon. 60 p. Off, nation. météorol. (climatologie) (mars).

OZENDA P... 1955. La température, facteur de répartition de la végétation en montagne. Ann. biol., 31, 5-6, 51-68.

PARdÉ J., 1958, Une belle réalisattion suédoise: la machine à mesurer les accroissements annuels des arbres. Rev. forest. franc., 4, 274-278.

PARDÉ J., 1959. Retour sur l'indice C.V.P. de Paterson. Rev. forest. franç., 1, 50-53.

PArdé J., 1961, Dendrométrie. Ec. nation. Eaux et Forêts, Nancy, 350 p.

ParDÉ J. 1963. Une méthode rapide de cubage approché des peuplements forestiers équiennes. Rev. forest. franç., 8-9, 714-720.

PARDÉ J., 1964. Indice climatique et production ligneuse. C.R. Acad, Agric. Fr., 569-576.

PARDÉ J., 1966. Waldbestände und Aufforstungen hober Wuchsleistung in Frankreich. Wiss. Ztschr. Techn. Univ. Dresden, 15, 2, 403-405.

PARDÉ J., 1968, Bases et méthodes d'évaluation de la productivité ligneuse stationnelle n'impliquant pas la coupe des arbres. 203-209, dans \& Proc. of the Copenhagen Symposium (Functioning of lerrestrial ecosystems at the primary production Level). 516 p. UNESCO, París.

PAtTERson S.S., 1956. The forest area of the World and its potential productivity. $216 \mathrm{p}$. Univ. Roy. Göteborg.

PERrin H., 1952. Sylviculture. Ec. nation. Eaux et Forêts Nancy, vol. I, 59-65.

Peyre de Fabregue B., 1962. Contribution à l'étude du milieu et de la végétation des Landes du massif de l'Aigoual. Thèse Fac. Sci. Univ. Montpellier.

Polge H., 1963. Contribution à l'étude de la qualité du bois des principales essences résineuses exotiques utilisées dans les reboisements français. Ann, Sci. forest., 20, 3 , 401-469.

Prodan M., 1965. Holzmesslehre, J.D, Sauerländer's Verlag, Frankfurt/Main, 644 p.

RoBl.IN M., 1961. Elements sur la rentabilité de lirrigation. Ann, agron., 12, 1, 65-73.

S.C.A.F.R., 1965. Etude du district-pilote de Reboisement de Saint-Martial, Ardèche. Société centrale d'aménagement foncier rural, 122, avenue de Neuilly, Neuilly-sur-Seine.

SCHÖPFER W., 1966. Automatisierung der Massen-Sorten - und Wertberechnung stehender Waldbestände. Schriftenr, Landesforstv. Baden-Wïrttemberg, 21, 108 p.

SiostrzonEK E., 1958. Radialzuwachs und Flächenzuwachs mit Bohrspänen und Stammscheiben Forstw. Cbl. 77, 7-8, 193-256.

SNEDECOR G.W., 1962. Statistical methods, $4^{*}$ éd, 534 p. Iowa State University Press, Ames, IOWA, USA.

Tomıssone R., 1967 a. Régression multiple progressive. Station de Biométrie. C.N.R.F. Programme FORTRAN 67.009.

Tomassone R., 1967 b. Une méthode d'investigation: la régression orthogonale. Ann. Sci. forest., 24, 3, 233-258.

Turc L., 1961. Evaluation des besoins en eau d'irrigation, évapotranspiration potentielle, formule simplifiće et mise à jour. Ann. agron., 12, 1, 13-49.

TURC L., 1967. Incidences des facteurs macroclimatiques sur les productions végétales. Fourrages, 31, 10-35.

VENET J., 1951. Découpes et classement. Rev, forest, franç., 4, 241-267. 